\title{
Synthesis, in vitro anti-inflammatory activity and molecular docking studies of novel 4,5-diarylthiophene-2-carboxamide derivatives
}

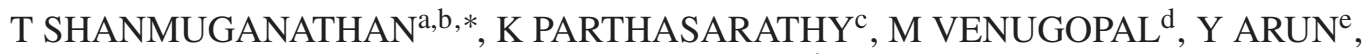 \\ N DHATCHANAMOORTHY ${ }^{\mathrm{a}}$ and A A M PRINCE ${ }^{\mathrm{b}}$ \\ ${ }^{a}$ Orchid Pharma Ltd, R \& D Centre, Chennai 600 119, India \\ ${ }^{b}$ Ramakrishna Mission Vivekananda College, Department of Chemistry, Mylapore, Chennai 600 004, India \\ ${ }^{c}$ Department of Chemistry, Siddha Central Research Institute, Central Council for Research in Siddha, \\ Chennai 600 106, India \\ ${ }^{\mathrm{d}}$ Ven Biotech Private Limited, Chennai, India \\ ${ }^{\text {e}}$ Organic Chemistry Division, Central Leather Research Institute (CSIR), Adyar, Chennai 600 020, India \\ Email: t_shanmuganathan09@yahoo.com
}

MS received 29 September 2016; revised 1 November 2016; accepted 12 November 2016

\begin{abstract}
A series of novel 4,5-diarylthiophene-2-carboxamide containing alkyl, cycloalkyl, aryl, aryl alkyl and heterocyclic alkyl moieties were synthesized, characterized and subsequently evaluated for antiinflammatory property. Among the novel compounds, the inhibition of bovine serum albumin denaturation assay revealed that the aryl and aryl alkyl derivatives of 4,5-diarylthiophene-2-carboxamide showed antiinflammatory activity comparable to the standard drug diclofenac sodium whereas alkyl and cycloalkyl amide derivatives showed less activity. Docking studies with these compounds against cyclooxygenase-2 receptor (PDB 1D: 1PXX) indicated that they exhibit specific interactions with key residues located in the site of the COX2 structure, which corroborates the hypothesis that these molecules are potential ligands of COX2. The analysis of the docking results, which takes into account the hydrophilic and hydrophobic interactions between the ligands and the target, identified $\mathrm{N}$-(4-bromophenyl)-4,5-bis(4-hydroxyphenyl)thiophene-2-carboxamide (6k) having high binding free energy of $-11.67 \mathrm{kcal} / \mathrm{mole}$ (comparable with standard diclofenac sodium) and the best docking score, indicating effective binding of the compound $\mathbf{6 k}$ at the active site.
\end{abstract}

Keywords. Demethylation; 4,5-diarylthiophene-2-carboxamide derivatives; anti-inflammatory; molecular docking.

\section{Introduction}

Non-steroidal anti-inflammatory drugs (NSAID) such as indomethacin and diclofenac are broadly used therapeutic drugs, primarily for pain, inflammation and arthritis. ${ }^{1}$ The beneficial anti-inflammatory and analgesic effects of NSAIDs is due to their inhibition of cyclooxygenase (COX) thereby inhibiting prostaglandins synthesis. ${ }^{2}$ Among the isoforms of COX, the inducible COX-2 is associated with inflammatory conditions whereas the constitutively expressed COX-1 is responsible for the cytoprotective effects of prostaglandins. The 2,3-diarylthiophene (DAT) pharmacophore is of importance following the report on the non-ulcerogenic, anti-inflammatory properties of 'DuP $697^{\prime}$ through the selective inhibition of COX-2. ${ }^{3}$ Further exploitation of DAT template resulted in the development of celecoxib, ${ }^{4}$ rofecoxib $^{5}$ and valdecoxib ${ }^{6}$ which are approved for clinical use.

\footnotetext{
*For correspondence
}

Desoxyanisoin (1) is one of the most versatile synthetic intermediates and widely utilized in the synthesis of complex heterocyclic compounds. Various bioactive molecules have been prepared by the efficient utilization of desoxyanisoin..$^{7-15}$ However, there are very few reports available in the literature on the synthesis of thiophene derivatives from desoxyanisoin. Considering the synthetic utility of desoxyanisoin, we envisaged to synthesize novel thiophene heterocycles, since sulphur possesses high therapeutic properties and its incorporation will substantially augment the biological activity.

The importance of cis-stilbene moiety in diaryl heterocyclic compounds is well-documented as responsible for receptor occupancy at the active site of COX enzyme ${ }^{16,17}$ whereas the nature of para-substituents on the aryl rings determine COX-2 selectivity. ${ }^{18,19}$ Further, the presence of methoxy group as an aryl substituent has been reported for the anti-inflammatory drugs such as Anitrazifen, Flumizole, Pamicogrel and Mofezolac whereas, free/derivatized amide group attached to the 
heterocyclic ring has been reported for COX inhibitors, such as Ethenzamide, Tenidap and Picotamide. This investigation is prompted mainly by the aforementioned anti-inflammatory activities of diarylheterocyclic ring system in addition to the recognized substituents to diaryl as well as heterocyclic moieties (Figure 1). In view of these observations, it was thought worthwhile to synthesise a series of 4,5-diarylthiophene-2-carboxamide derivatives via Vilsmeier procedure followed by the incorporation with 2-mercaptoacetic acid to yield substituted thiophenes and to evaluate them for their anti-inflammatory activity.

\section{Experimental}

\subsection{Materials and methods}

All the chemicals and reagents used were lab grade material procured from Alfa aesar. All the solvents used were purchased from commercial suppliers and were used without further purification. The melting points were determined using Buchi apparatus by the open capillary tube method and are uncorrected. The IR spectra were recorded in PerkinElmer series 2000 FTIR spectrophotometer using $\mathrm{KBr}$ pellet. ${ }^{1} \mathrm{H}$ NMR and ${ }^{13} \mathrm{C}$ NMR spectra were obtained in $\mathrm{CDCl}_{3}$, DMSO- $d_{6}$ on a Bruker spectrometer at 400 and $100 \mathrm{MHz}$, respectively. The chemical shifts are reported in ppm $(\delta)$ relative to tetramethylsilane as internal standard, coupling constants $(J)$ are in hertz $(\mathrm{Hz})$. Spin multiplicities are given as s (singlet), d (doublet), $t$ (triplet), dd (doublet of doublet), bs (broad signal) and $\mathrm{m}$ (multiplet). Residual proton and carbon solvent signal for $\mathrm{CDCl}_{3}, \delta_{\mathrm{H}} 7.26 \mathrm{ppm}, \delta_{\mathrm{C}} 77.0 \mathrm{ppm}$, $\delta_{6}$-DMSO, $\delta_{\mathrm{H}} 2.50 \mathrm{ppm}, \delta_{\mathrm{C}} 40.0 \mathrm{ppm}$. Proton and carbon spectra were typically obtained at room temperature. Mass spectra were recorded on ESI - Perkin Elmer Sciex, API 3000 mass spectrometer. Pre-coated silica gel GF254 plates from Merck were used for thin layer chromatography (TLC). The elemental analyses were recorded in Thermo Finnigan Flash EA 1112 elemental analyser.

\subsection{Synthesis}

2.2a Synthesis of 2,3-bis (4-methoxy phenyl)-3-chloro-2prop-2-ene aldehyde (2): $0.05 \mathrm{~mol}$ (7.66 g) of phosphorous oxychloride $\left(\mathrm{POCl}_{3}\right)$ was added drop wise over a period of $15-30 \mathrm{~min}$ with stirring at $0-5^{\circ} \mathrm{C}$ to $20 \mathrm{~mL}$ of dimethylformamide. The mass was maintained at $0-5^{\circ} \mathrm{C}$ over $30 \mathrm{~min}$ and a solution of desoxyanisoin $0.04 \mathrm{~mol}(10 \mathrm{~g})$ in dimethylformamide $(50 \mathrm{~mL})$ at $0-5^{\circ} \mathrm{C}$ was added under stirring. The reaction mass was heated to $70-75^{\circ} \mathrm{C}$ and maintained for $4 \mathrm{~h}$. The progress of the reaction was monitored using TLC (Toluene). After completion, the reaction mixture was cooled and poured slowly into $25 \%$ solution of sodium acetate in water $(100 \mathrm{~mL})$. The product was filtered and washed with water, followed by slurry wash with ethanol $(100 \mathrm{~mL})$ which after drying gave $9.1 \mathrm{~g}$ (78\% yield) of the compound 2 .

White powder, M.p. $145-147^{\circ} \mathrm{C}$. [ Lit. $^{20} ; 158^{\circ} \mathrm{C}$ ]. IR $(\mathrm{KBr})$ $\mathrm{cm}^{-1}: 2932,1680,1513,1442,1080,813,772 .{ }^{1} \mathrm{H}$ NMR $\left(400 \mathrm{MHz}, \mathrm{CDCl}_{3}\right): \delta 9.66(\mathrm{~s}, 1 \mathrm{H}), 7.52(\mathrm{~d}, J=7.8 \mathrm{~Hz}, 2 \mathrm{H})$, 7.25-7.22 (m, 2H), 6.99-6.91 (m, 4H), 3.89 (s, 3H), 3.85 (s, $3 \mathrm{H}) .{ }^{13} \mathrm{C}$ NMR $\left(100 \mathrm{MHz}, \mathrm{CDCl}_{3}\right): \delta 190.5,162.1,159.7$, 155.1, 139.8, 132.4, 132.1, 131.1, 130.6, 128.4, 126.7, 114.2, 113.9, 55.8, 55.5. ESI-MS m/z Calculated 302.1. Found: $303.1[\mathrm{M}+\mathrm{H}]^{+}$.

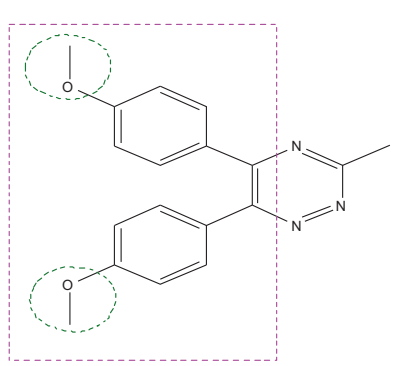

Anitrazifen

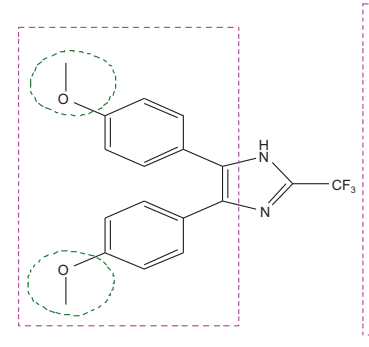

Flumizole

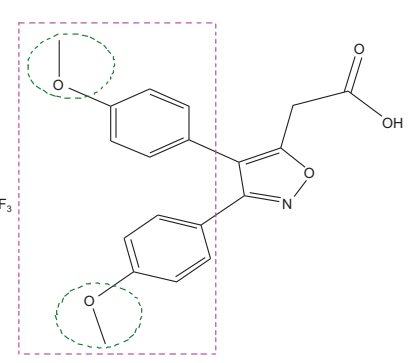

Mofezolac

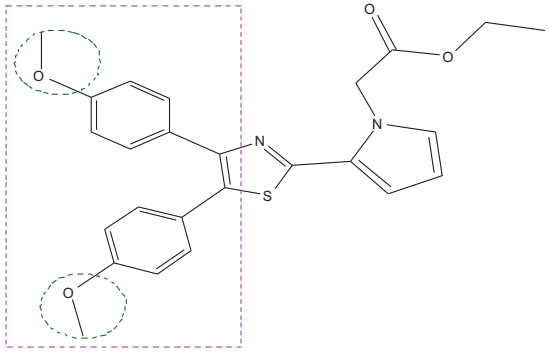

Pamicogrel

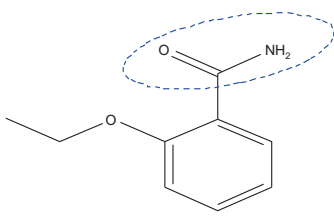

Ethenzamide

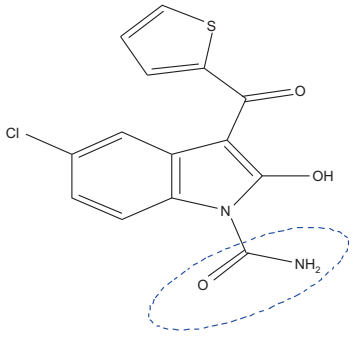

Tenidap

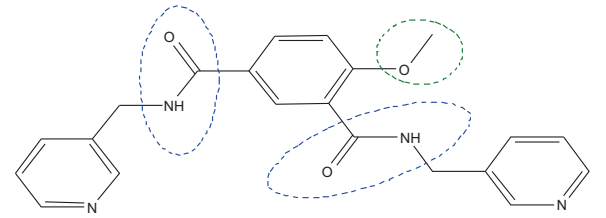

Picotamide

Figure 1. Anti-inflammatory agents based on diaryl heterocyclic and amide based compounds. 
2.2b Synthesis of 4,5-bis(4-methoxyphenyl)thiophene-2carboxylic acid (3): 2,3-bis (4-methoxy phenyl)-3-chloro2-prop-2-ene aldehyde 2 (5 g, 0.0165 mole) was added to a solution of potassium hydroxide ( $4 \mathrm{~g}, 0.714$ mole) and 2mercapto acetic acid (3.1 g, 0.034 mole) in methanol:water (40 mL:10 mL) mixture at room temperature. The mixture was refluxed for $4 \mathrm{~h}$, and reaction was monitored using TLC (hexane: ethyl acetate 3:7). After completion, the reaction mixture was cooled to room temperature and slowly acidified with concentrated $\mathrm{HCl}$ over $30-45 \mathrm{~min}$ at $25-30^{\circ} \mathrm{C}$. The product 3 was filtered, washed with water and dried to get compound 3. $4 \mathrm{~g}$ (71\% yield).

Yellow powder, M.p. $211-213^{\circ} \mathrm{C}$. [Lit. ${ }^{20} ; 215{ }^{\circ} \mathrm{C}$ ]. IR (KBr) cm ${ }^{-1}:$ 2933, 2542, 1668, 1546, 1449, 1247, 1033, 827. ${ }^{1} \mathrm{H}$ NMR (400 MHz, $\left.\mathrm{CDCl}_{3}\right): \delta 10.7$ (bs, $\left.1 \mathrm{H}\right), 7.87(\mathrm{~s}, 1 \mathrm{H})$, 7.27 (d, $J=8.6 \mathrm{~Hz}, 2 \mathrm{H}), 7.20$ (d, $J=8.6 \mathrm{~Hz}, 2 \mathrm{H}), 6.85-6.81$ $(\mathrm{m}, 4 \mathrm{H}), 3.83(\mathrm{~s}, 3 \mathrm{H}), 3.81(\mathrm{~s}, 3 \mathrm{H}) .{ }^{13} \mathrm{C}$ NMR $(100 \mathrm{MHz}$, $\left.\mathrm{CDCl}_{3}\right): \delta 168.0,159.9,159.1,147.0,138.4,137.9,130.6$, $130.3,129.5128 .1,125.9,114.3,114.1,55.5,55.4$. ESI-MS $\mathrm{m} / \mathrm{z}$ Calculated 340.1. Found: $339.1[\mathrm{M}-\mathrm{H}]^{-}$.

2.2c Synthesis of 4,5-bis(4-hydroxyphenyl)thiophene-2carboxylic acid (4): 4,5-bis(4-methoxyphenyl)thiophene2-carboxylic acid 3 (6 g, 0.0176 mole) was added to the mixture of aluminium chloride (9.38 g, 0.0704 mole) in chlorobenzene $(60 \mathrm{~mL})$ at $25-30^{\circ} \mathrm{C}$. The reaction mixture was heated to $95-105^{\circ} \mathrm{C}$ and maintained for $3 \mathrm{~h}$. The progress of the reaction was monitored using TLC (hexane: ethyl acetate 3:7). After completion of the reaction, aqueous $\mathrm{HCl}$ $(1: 1,50 \mathrm{~mL})$ was added to the mass at $25-30^{\circ} \mathrm{C}$. The product was extracted with ethyl acetate $(50 \mathrm{~mL} \times 2)$ and the organic layer was washed with water. Ethyl acetate was evaporated under vacuum and the residue was triturated with dichloromethane $(100 \mathrm{~mL})$, filtered to get compound 4. $4.85 \mathrm{~g}$. (88\% yield).

Light green powder, M.p. $240-243^{\circ} \mathrm{C}$. IR $(\mathrm{KBr}) \mathrm{cm}^{-1}$ : $3428,3318,1639,1545,1442,1255,1069,829 .{ }^{1} \mathrm{H}$ NMR $\left(400 \mathrm{MHz}, \mathrm{CDCl}_{3}+\mathrm{DMSO}_{6}\right): \delta 8.48$ (bs, $\left.1 \mathrm{H}\right), 8.19$ (bs, $1 \mathrm{H}), 7.75$ (s, 1H), 7.17 (d, $J=8.5 \mathrm{~Hz}, 2 \mathrm{H}), 7.11(\mathrm{~d}, J=8.4$ $\mathrm{Hz}, 2 \mathrm{H}), 6.77-6.75$ (m, 4H). ${ }^{13} \mathrm{C}$ NMR (100 MHz, $\mathrm{CDCl}_{3}+$ DMSO-d 6 ): $\delta 164.3,157.5,156.4,144.9,137.8,136.2$, 130.9, 130.4, 130.0, 127.1, 124.9, 115.6, 115.5. ESI-MS m/z Calculated 312.0. Found: $313.0[\mathrm{M}+\mathrm{H}]^{+}$.

$2.2 \mathrm{~d}$ General synthetic procedure for the title compounds 4,5-bis(4-methoxyyphenyl)-substituedthiophene-2-carboxamide $(\mathbf{5} \boldsymbol{a}-\mathbf{5 o})$ : Dimethylaminopyridine $(1.2 \mathrm{mmol})$ was added to

Table 1. Reaction time and yield of the product.

\begin{tabular}{|c|c|c|c|c|c|}
\hline Entry & $\mathrm{R}$ & $\mathrm{R}^{1}$ & Time (h) & Product $^{\mathrm{a}}$ & Yield $^{\mathrm{b}}(\%)$ \\
\hline$\overline{1}$ & $\mathrm{CH}_{3}$ & Propyl & 3 & $5 a$ & 76 \\
\hline 2 & $\mathrm{CH}_{3}$ & isopropyl & 7 & $5 b$ & 78 \\
\hline 3 & $\mathrm{CH}_{3}$ & butyl & 4 & $5 c$ & 80 \\
\hline 4 & $\mathrm{CH}_{3}$ & cyclopropyl & 3 & $5 d$ & 75 \\
\hline 5 & $\mathrm{CH}_{3}$ & cyclopentyl & 3 & $5 e$ & 76 \\
\hline 6 & $\mathrm{CH}_{3}$ & cyclohexyl & 3 & $5 f$ & 75 \\
\hline 7 & $\mathrm{CH}_{3}$ & cycloheptyl & 3 & $5 g$ & 72 \\
\hline 8 & $\mathrm{CH}_{3}$ & Phenyl & 5 & $5 \mathrm{~h}$ & 85 \\
\hline 9 & $\mathrm{CH}_{3}$ & 4-fluorophenyl & 4 & $5 \mathrm{i}$ & 90 \\
\hline 10 & $\mathrm{CH}_{3}$ & 4-chlorophenyl & 4 & $5 \mathrm{j}$ & 88 \\
\hline 11 & $\mathrm{CH}_{3}$ & 4-bromophenyl & 6 & $5 \mathrm{k}$ & 87 \\
\hline 12 & $\mathrm{CH}_{3}$ & benzyl & 4 & 51 & 80 \\
\hline 13 & $\mathrm{CH}_{3}$ & 4-fluorobenzyl & 4 & $5 \mathrm{~m}$ & 85 \\
\hline 14 & $\mathrm{CH}_{3}$ & 4-(trifluoromethyl)benzyl & 4 & $5 n$ & 86 \\
\hline 15 & $\mathrm{CH}_{3}$ & 2-(thiophene-2-yl)ethyl & 4.5 & 50 & 90 \\
\hline 16 & $\mathrm{H}$ & Propyl & 5 & $6 a$ & 79 \\
\hline 17 & $\mathrm{H}$ & isopropyl & 5 & $6 b$ & 80 \\
\hline 18 & $\mathrm{H}$ & butyl & 5 & $6 c$ & 75 \\
\hline 19 & $\mathrm{H}$ & cyclopropyl & 5 & $6 d$ & 85 \\
\hline 20 & $\mathrm{H}$ & cyclopentyl & 5 & $6 e$ & 89 \\
\hline 21 & $\mathrm{H}$ & cyclohexyl & 5 & $6 f$ & 86 \\
\hline 22 & $\mathrm{H}$ & cycloheptyl & 5 & $6 g$ & 82 \\
\hline 23 & $\mathrm{H}$ & Phenyl & 3 & $6 \mathrm{~h}$ & 80 \\
\hline 24 & $\mathrm{H}$ & 4-fluorophenyl & 3 & $6 \mathrm{i}$ & 82 \\
\hline 25 & $\mathrm{H}$ & 4-chlorophenyl & 6 & $6 j$ & 78 \\
\hline 26 & $\mathrm{H}$ & 4-bromophenyl & 6 & $6 \mathrm{k}$ & 76 \\
\hline 27 & $\mathrm{H}$ & Benzyl & 5 & 61 & 95 \\
\hline 28 & $\mathrm{H}$ & 4-fluorobenzyl & 5 & $6 \mathrm{~m}$ & 93 \\
\hline 29 & $\mathrm{H}$ & 4-(trifluoromethyl)benzyl & 5 & $6 n$ & 90 \\
\hline 30 & $\mathrm{H}$ & 2-(thiophene-2-yl)ethyl & 4 & 60 & 80 \\
\hline
\end{tabular}

${ }^{\mathrm{a}}$ All the products were characterized by NMR, IR and Mass spectroscopy; ${ }^{\mathrm{b}}$ Isolated yield. 
a mixture of 4,5-bis(4-methoxyphenyl)thiophene-2-carboxylic acid (3) (1.0 mmol), 2-(1H-Benzotriazole-1-yl)-1,1,3,3tetramethyluronium tetrafluoroborate (TBTU) $(1.2 \mathrm{mmol})$ in acetonitrile $(3 \mathrm{~mL})$ at $25-30^{\circ} \mathrm{C}$ and stirred for $30 \mathrm{~min}$. An appropriate amine $(1.2 \mathrm{mmol})$ was added to the reaction mixture at $25-30^{\circ} \mathrm{C}$ and progress of the reaction was monitored by TLC. (hexane: ethyl acetate: acetic acid $4 \mathrm{~mL}$ : $4 \mathrm{~mL}: 2$ drops). After completion of the reaction, the mixture was poured into water and the product was extracted with ethyl acetate. The organic layer was washed with 5\% aqueous $\mathrm{HCl}$ solution, followed by a saturated solution of sodium bicarbonate and finally with water. The organic layer was separated and dried over anhydrous sodium sulphate. The solvent was evaporated under vacuum to get the product. The reaction times and yields of the product are shown in Table 1.

\section{$2.2 \mathrm{~d} 1$ 4,5-bis(4-methoxyphenyl)-N-propylthiophene-}

2-carboxamide (5a): Prepared from 3 and propyl amine. Off-white powder, M.p. $56-59^{\circ} \mathrm{C}$. IR $(\mathrm{KBr}) \mathrm{cm}^{-1}$ : 3306, 2931, 2835, 1622, 1556, 1247, 1032, 829, 794. ${ }^{1} \mathrm{H}$ NMR $\left(400 \mathrm{MHz}, \mathrm{DMSO}-\mathrm{d}_{6}\right): \delta 8.50(\mathrm{t}, J=5.6 \mathrm{~Hz}, 1 \mathrm{H}), 7.83(\mathrm{~s}$, $1 \mathrm{H}), 7.23-7.16(\mathrm{~m}, 4 \mathrm{H}), 6.93-6.89(\mathrm{~m}, 4 \mathrm{H}), 3.76(\mathrm{~s}, 3 \mathrm{H})$, 3.75 (s, 3H), 3.24 (quartet, $J=6.6 \mathrm{~Hz}, 2 \mathrm{H}$ ), 1.58 (sextet, $J=7.4 \mathrm{~Hz}, 2 \mathrm{H}), 0.92(\mathrm{t}, J=7.4 \mathrm{~Hz}, 3 \mathrm{H}) .{ }^{13} \mathrm{C}$ NMR $(100$ MHz, DMSO-d ${ }_{6}$ ): $\delta 160.9,159.2,158.4,140.9,137.4$, $137.1,130.6,130.1,129.8,127.9,125.6,114.3,114.0$, 55.2, 55.1, 40.9, 22.5, 11.5. ESI-MS m/z Calculated 381.1. Found: $382.1[\mathrm{M}+\mathrm{H}]^{+}$. Anal. Calculated for $\mathrm{C}_{22} \mathrm{H}_{23} \mathrm{NO}_{3} \mathrm{~S}$ : C, 69.26; H, 6.08; N, 3.67\%. Found: C, 69.12; H, 6.00; N, $3.52 \%$.

2.2d2 N-isopropyl-4,5-bis(4-methoxyphenyl)thiophene2-carboxamide (5b): Prepared from 3 and isopropyl amine. White powder, M.p. $222-225^{\circ} \mathrm{C}$. IR $(\mathrm{KBr}) \mathrm{cm}^{-1}$ : $3292,2931,2837,1620,1558,1248,1032,836,791 .{ }^{1} \mathrm{H}$ NMR (400 MHz, DMSO-d 6 ): $\delta 8.28(\mathrm{~d}, J=7.8 \mathrm{~Hz}, 1 \mathrm{H})$, $7.88(\mathrm{~s}, 1 \mathrm{H}), 7.22-7.17(\mathrm{~m}, 4 \mathrm{H}), 6.93-6.89(\mathrm{~m}, 4 \mathrm{H}), 4.08$ (septet, $J=6.8 \mathrm{~Hz}, 1 \mathrm{H}), 3.76(\mathrm{~s}, 3 \mathrm{H}), 3.75(\mathrm{~s}, 3 \mathrm{H}), 1.19(\mathrm{~d}$, $J=6.4 \mathrm{~Hz}, 6 \mathrm{H}) .{ }^{13} \mathrm{C}$ NMR $(100 \mathrm{MHz}$, DMSO-d 6 ): $\delta 160.0$, $159.2,158.4,140.9,137.6,137.1,130.7,130.1,129.8$, $127.9,125.7,114.3,114.0,55.2,55.1,41.1,22.4$. ESI-MS $\mathrm{m} / \mathrm{z}$ Calculated 381.1. Found: $382.1[\mathrm{M}+\mathrm{H}]^{+}$. Anal. Calculated for $\mathrm{C}_{22} \mathrm{H}_{23} \mathrm{NO}_{3} \mathrm{~S}$ : C, 69.26; $\mathrm{H}, 6.08 ; \mathrm{N}, 3.67 \%$. Found: C, 69.32; H, 6.14; N, 3.56\%.

$2.2 \mathrm{~d} 3 \quad \mathrm{~N}$-butyl-4,5-bis(4-methoxyphenyl)thiophene-2carboxamide (5c): Prepared from 3 and n-butyl amine. Off-white powder. M.p. $53-55^{\circ} \mathrm{C}$. IR $(\mathrm{KBr}) \mathrm{cm}^{-1}$ : 3303, 2931, 2835, 1609, 1556, 1248, 1032, 829, 795. ${ }^{1} \mathrm{H}$ NMR (400 MHz, DMSO-d $\left.{ }_{6}\right): \delta 8.48(\mathrm{t}, J=5.6 \mathrm{~Hz}, 1 \mathrm{H}), 7.82(\mathrm{~s}, 1 \mathrm{H})$, 7.22-7.17 (m, 4H), 6.93-6.89 (m, 4H), $3.76(\mathrm{~s}, 3 \mathrm{H}), 3.75(\mathrm{~s}$, $3 \mathrm{H}$ ), 3.27 (quartet, $J=6.8 \mathrm{~Hz}, 2 \mathrm{H}$ ), 1.54 (quintet, $J=6.9 \mathrm{~Hz}$, $2 \mathrm{H}$ ), 1.38 (sextet, $J=7.4 \mathrm{~Hz}, 2 \mathrm{H}$ ), 0.92 (t, $J=7.3 \mathrm{~Hz}, 3 \mathrm{H}$ ). ${ }^{13} \mathrm{C}$ NMR $(100 \mathrm{MHz}$, DMSO-d 6 ): $\delta 160.8,159.2,158.4$, $140.9,137.4,137.1,130.6,130.1,129.8,127.9,125.6$, $114.3,114.0,55.2,55.1,40.2,31.3,19.6,13.7$. ESI-MS m/z Calculated 395.1. Found: $396.1[\mathrm{M}+\mathrm{H}]^{+}$. Anal. Calculated for $\mathrm{C}_{23} \mathrm{H}_{25} \mathrm{NO}_{3} \mathrm{~S}$ : C, 69.84; H, 6.36; N, 3.54\%. Found: C, $69.48 ; \mathrm{H}, 6.24 ; \mathrm{N}, 3.56 \%$.

$2.2 \mathrm{~d} 4 \mathrm{~N}$-cyclopropyl-4,5-bis(4-methoxyphenyl)thiophene2-carboxamide $(\mathbf{5} \boldsymbol{d})$ : $\quad$ Prepared from 3 and cyclopropyl amine. Pale brown powder, M.p. $160-162^{\circ} \mathrm{C}$. IR $(\mathrm{KBr})$ $\mathrm{cm}^{-1}$ : 3349, 2931, 2836, 1626, 1550, 1249, 1036, 832, 798. ${ }^{1} \mathrm{H}$ NMR (400 MHz, DMSO-d 6 ): $\delta 8.49(\mathrm{~d}, J=4 \mathrm{~Hz}, 1 \mathrm{H})$, $7.79(\mathrm{~s}, 1 \mathrm{H}), 7.21-7.1(\mathrm{~m}, 4 \mathrm{H}), 6.92-6.89(\mathrm{~m}, 4 \mathrm{H}), 3.76$ (s, 3H), $3.75(\mathrm{~s}, 3 \mathrm{H}), 2.84-2.79(\mathrm{~m}, 1 \mathrm{H}), 0.73-0.69(\mathrm{~m}$, 2H), 0.58-0.54 (m, 2H). ${ }^{13} \mathrm{C}$ NMR (100 MHz, DMSO-d 6 ): $\delta 162.1,159.2,158.4,141.1,137.1,130.8,130.1,129.8$, 127.9, 125.6, 114.3, 114.0, 55.2, 55.1, 22.9, 5.8. ESI-MS $\mathrm{m} / \mathrm{z}$ Calculated 379.1. Found: $380.1[\mathrm{M}+\mathrm{H}]^{+}$. Anal. Calculated for $\mathrm{C}_{22} \mathrm{H}_{21} \mathrm{NO}_{3} \mathrm{~S}: \mathrm{C}, 69.63 ; \mathrm{H}, 5.58 ; \mathrm{N}, 3.69 \%$. Found: C, 69.52; H, 5.48; N, 3.58\%.

2.2d5 N-cyclopentyl-4,5-bis(4-methoxyphenyl)thiophene2-carboxamide (5e): Prepared from 3 and cyclopentyl amine. Off-white powder, M.p. $193-195^{\circ} \mathrm{C}$. IR $(\mathrm{KBr}) \mathrm{cm}^{-1}$ : $3244,2952,2834,1613,1557,1249,1031,829,795 .{ }^{1} \mathrm{H}$ NMR (400 MHz, DMSO-d $\left.\mathrm{d}_{6}\right): \delta 8.32(\mathrm{~d}, J=7.3 \mathrm{~Hz}, 1 \mathrm{H})$, $7.89(\mathrm{~s}, 1 \mathrm{H}), 7.22-7.17(\mathrm{~m}, 4 \mathrm{H}), 6.92-6.89(\mathrm{~m}, 4 \mathrm{H}), 4.22-$ $4.17(\mathrm{~m}, 1 \mathrm{H}), 3.76(\mathrm{~s}, 3 \mathrm{H}), 3.75(\mathrm{~s}, 3 \mathrm{H}), 1.90-1.86(\mathrm{~m}$, $2 \mathrm{H}), 1.69(\mathrm{~m}, 2 \mathrm{H}), 1.56-1.49(\mathrm{~m}, 4 \mathrm{H}) .{ }^{13} \mathrm{C}$ NMR $(100 \mathrm{MHz}$, DMSO- $\left.\mathrm{d}_{6}\right): \delta 160.5,159.2,158.4,140.9,137.5,137.1$, 130.8, 130.1, 129.8, 127.9, 125.6, 114.3, 114.0, 55.2, 55.1, 50.9, 32.2, 23.7. ESI-MS m/z Calculated 407.1. Found: $408.1[\mathrm{M}+\mathrm{H}]^{+}$. Anal. Calculated for $\mathrm{C}_{24} \mathrm{H}_{25} \mathrm{NO}_{3} \mathrm{~S}$ : C, 70.73; H, 6.18; N, 3.44\%. Found: C, 70.69; H, 6.28; N, $3.48 \%$.

\section{2d6 N-cyclohexyl-4,5-bis(4-methoxyphenyl)}

thiophene-2-carboxamide (5f): Prepared from 3 and cyclohexyl amine. White powder, M.p. $206-207^{\circ} \mathrm{C}$. IR (KBr) $\mathrm{cm}^{-1}: 3236,2928,2852,1627,1563,1244,1034$, 833, 796. ${ }^{1} \mathrm{H}$ NMR $(400 \mathrm{MHz}$, DMSO-d 6 ): $\delta 8.25(\mathrm{~d}, J=$ $7.9 \mathrm{~Hz}, 1 \mathrm{H}), 7.88(\mathrm{~s}, 1 \mathrm{H}), 7.22-7.17$ (m, 4H), 6.92-6.89 (m, $4 \mathrm{H}), 3.76(\mathrm{~m}, 7 \mathrm{H}), 1.84(\mathrm{~m}, 2 \mathrm{H}), 1.73(\mathrm{~m}, 2 \mathrm{H}), 1.62-1.59$ (m, 1H), 1.29-1.27 (m, 4H), 1.14-1.12 (m, 1H). ${ }^{13} \mathrm{C}$ NMR $\left(100 \mathrm{MHz}, \mathrm{DMSO}-\mathrm{d}_{6}\right): \delta 160.0,159.2,158.4,140.9,137.6$, $137.1,130.7,130.1,129.8,127.9,125.7,114.3,114.0,55.2$, 55.1, 48.4, 32.5, 25.3, 24.9. ESI-MS m/z Calculated 421.2. Found: $422.2[\mathrm{M}+\mathrm{H}]^{+}$. Anal. Calculated for $\mathrm{C}_{25} \mathrm{H}_{27} \mathrm{NO}_{3} \mathrm{~S}$ : C, 71.23; H, 6.46; N, 3.32\%. Found: C, 71.46; H, 6.38; N, $3.28 \%$.

\section{2d7 N-cycloheptyl-4,5-bis(4-methoxyphenyl)}

thiophene-2-carboxamide (5g): Prepared from 3 and cycloheptyl amine. White powder, M.p. $212-214^{\circ} \mathrm{C}$. IR $(\mathrm{KBr}) \mathrm{cm}^{-1}$ : 3243, 2925, 2854, 1609, 1562, 1244, 1037, 830, 795. ${ }^{1} \mathrm{H}$ NMR (400 MHz, DMSO-d $\left.\mathrm{d}_{6}\right): \delta 8.29(\mathrm{~d}, J=$ $7.9 \mathrm{~Hz}, 1 \mathrm{H}), 7.89$ (s, 1H), 7.21-7.17 (m, 4H), 6.92-6.89 (m, $4 \mathrm{H}), 3.95-3.91(\mathrm{~m}, 1 \mathrm{H}), 3.76(\mathrm{~s}, 3 \mathrm{H}), 3.75(\mathrm{~s}, 3 \mathrm{H}), 1.89-1.85$ (m, 2H), 1.67-1.63 (m, 2H), 1.58-1.51 (m, 5H), 1.49-1.39 $(\mathrm{m}, 3 \mathrm{H}) .{ }^{13} \mathrm{C}$ NMR $\left(100 \mathrm{MHz}\right.$, DMSO-d $\left.\mathrm{d}_{6}\right): \delta 159.8,159.2$, 158.4, 140.9, 137.7, 137.1, 130.7, 130.1, 129.8, 128.0, 125.7, 
114.3, 114.0, 55.2, 55.1, 50.5, 34.4, 27.9, 23.9. ESI-MS m/z Calculated 435.2. Found: $436.2[\mathrm{M}+\mathrm{H}]^{+}$. Anal. Calculated for $\mathrm{C}_{26} \mathrm{H}_{29} \mathrm{NO}_{3} \mathrm{~S}$ : C, 71.69; H, 6.71; N, 3.22\%. Found: C, $71.66 ; \mathrm{H}, 6.68 ; \mathrm{N}, 3.26 \%$.

\section{2d8 4,5-bis(4-methoxyphenyl)-N-phenylthiophene-} 2-carboxamide (5h): Prepared from $\mathbf{3}$ and aniline. Pale yellow powder, M.p. $158-160^{\circ} \mathrm{C}$. IR $(\mathrm{KBr}) \mathrm{cm}^{-1}$ : 3308 , 2930, 2833, 1632, 1551, 1246, 1033, 830, 754. ${ }^{1} \mathrm{H}$ NMR (400 MHz, DMSO-d 6 ): $\delta 10.24(\mathrm{~s}, 1 \mathrm{H}), 8.13$ (s, 1H), 7.76 $(\mathrm{d}, J=7.8 \mathrm{~Hz}, 2 \mathrm{H}), 7.39(\mathrm{t}, J=7.8 \mathrm{~Hz}, 2 \mathrm{H}), 7.26-7.22(\mathrm{~m}$, $4 \mathrm{H}), 7.13(\mathrm{t}, J=7.4 \mathrm{~Hz}, 1 \mathrm{H}), 6.95-6.92(\mathrm{~m}, 4 \mathrm{H}), 3.77(\mathrm{~s}$, $3 \mathrm{H}), 3.75$ (s, 3H). ${ }^{13} \mathrm{C}$ NMR (100 MHz, DMSO-d 6 ): $\delta 159.7$, $159.4,158.6,142.3,138.8,137.4,137.1,131.9,130.2$, 129.9, 128.8, 127.8, 125.5, 123.8, 120.2, 114.4, 114.1, 55.3, 55.2. ESI-MS m/z Calculated 415.1. Found: $416.2[\mathrm{M}+$ $\mathrm{H}]^{+}$. Anal. Calculated for $\mathrm{C}_{25} \mathrm{H}_{21} \mathrm{NO}_{3} \mathrm{~S}$ : C, 72.27; H, 5.09; N, 3.37\%. Found: C, 72.41; H, 5.06; N, 3.34\%.

\section{2d9 N-(4-fluorophenyl)-4,5-bis(4-methoxyphenyl)} thiophene-2-carboxamide (5i): Prepared from 3 and 4fluoroaniline. Off-white powder, M.p. $176-178^{\circ} \mathrm{C}$. IR $(\mathrm{KBr})$ $\mathrm{cm}^{-1}$ : 3312, 2931, 2834, 1632, 1557, 1246, 1032, 830, 795.

${ }^{1} \mathrm{H}$ NMR (400 MHz, DMSO-d 6 ): $\delta 10.30(\mathrm{~s}, 1 \mathrm{H}), 8.10(\mathrm{~s}$, 1H), 7.78-7.75 (m, 2H), 7.26-7.19 (m, 6H), 6.95-6.92 (dd, $4 \mathrm{H}, J=8.8 \mathrm{~Hz} \& J=3.1 \mathrm{~Hz}), 3.77(\mathrm{~s}, 6 \mathrm{H}) .{ }^{13} \mathrm{C} \mathrm{NMR}$ $\left(100 \mathrm{MHz}, \mathrm{DMSO}-\mathrm{d}_{6}\right): \delta$ 159.6, 159.5, 159.4, 158.6, 157.2, $142.4,137.4,136.9,135.2,135.1,131.9,130.2,129.9$, 127.8, 125.4, 122.1, 122.0, 115.5, 115.3, 114.4, 114.1, 55.3, 55.1. ESI-MS m/z Calculated 433.1. Found: 434.1 [M + $\mathrm{H}]^{+}$. Anal. Calculated for $\mathrm{C}_{25} \mathrm{H}_{20} \mathrm{FNO}_{3} \mathrm{~S}$ : C, 69.27; H, 4.65; N, 3.23\%. Found: C, 69.10; H, 4.45; N, 3.12\%.

2.2d10 N-(4-chlorophenyl)-4,5-bis(4-methoxyphenyl) thiophene-2-carboxamide (5j): Prepared from $\mathbf{3}$ and 4-chloroaniline. Pale yellow powder, M.p. $152-154^{\circ} \mathrm{C}$. IR $(\mathrm{KBr}) \mathrm{cm}^{-1}$ : 3358, 2931, 2834, 1636, 1582, 1248, 1033, 827, 795. ${ }^{1} \mathrm{H}$ NMR (400 MHz, DMSO-d 6 ): $\delta 10.36(\mathrm{~s}$, $1 \mathrm{H}), 8.11(\mathrm{~s}, 1 \mathrm{H}), 7.79(\mathrm{~d}, J=8.9 \mathrm{~Hz}, 2 \mathrm{H}), 7.44(\mathrm{~d}, J=$ $8.8 \mathrm{~Hz}, 2 \mathrm{H}), 7.26(\mathrm{t}, J=8.5 \mathrm{~Hz}, 4 \mathrm{H}), 6.95-6.92(\mathrm{dd}, J=$ $8.8 \mathrm{~Hz} \& J=2.7 \mathrm{~Hz}, 4 \mathrm{H}), 3.77(\mathrm{~s}, 3 \mathrm{H}), 3.75(\mathrm{~s}, 3 \mathrm{H}) .{ }^{13} \mathrm{C}$ NMR (100MHz, DMSO-d 6$): \delta 159.8,159.4,158.6,142.6$, $137.8,137.5,136.7,132.2,130.2,129.9,128.7,127.7$, 127.4, 125.4, 121.7, 114.4, 114.1, 55.3, 55.2. ESI-MS m/z Calculated 449.0. Found: $448.0[\mathrm{M}-\mathrm{H}]^{-}$. Anal. Calculated for $\mathrm{C}_{25} \mathrm{H}_{20} \mathrm{ClNO}_{3} \mathrm{~S}$ : C, 66.73; H, 4.48; N, 3.11\%. Found: C, $67.10 ; \mathrm{H}, 4.25 ; \mathrm{N}, 2.88 \%$.

2.2d11 N-(4-bromophenyl)-4,5-bis(4-methoxyphenyl) thiophene-2-carboxamide (5k): Prepared from $\mathbf{3}$ and 4-bromoaniline. Pale yellow powder, M.p. $170-172^{\circ} \mathrm{C}$. IR $(\mathrm{KBr}) \mathrm{cm}^{-1}$ : 3353, 2931, 2834, 1638, 1548, 1248, 1034, 837, 727. ${ }^{1} \mathrm{H}$ NMR (400 MHz, DMSO-d 6 ): $\delta 10.35$ (s, 1H), $8.11(\mathrm{~s}, 1 \mathrm{H}), 7.74$ (d, $J=8.9 \mathrm{~Hz}, 2 \mathrm{H}), 7.57$ (d, $J=8.72$ $\mathrm{Hz}, 2 \mathrm{H}), 7.26-7.22(\mathrm{~m}, 4 \mathrm{H}), 6.95-6.92(\mathrm{~m}, 4 \mathrm{H}), 3.77$ (s, $3 \mathrm{H}), 3.75$ (s, 3H). ${ }^{13} \mathrm{C}$ NMR (100MHz, DMSO-d 6 ): $\delta 159.8$, 159.4, 158.6, 142.6, 138.2, 137.5, 136.7, 132.2, 131.6, 130.2,
129.9, 127.7, 125.4, 122.1, 115.5, 114.4, 114.1, 55.3, 55.2. ESI-MS m/z Calculated 493.0. Found: $492.0\left[{ }_{79} \mathrm{M}^{\mathrm{Br}}-\mathrm{H}\right]^{-}$; $493.9\left[{ }_{81} \mathrm{M}^{\mathrm{Br}}-\mathrm{H}\right]^{-}$. Anal. Calculated for $\mathrm{C}_{25} \mathrm{H}_{20} \mathrm{BrNO}_{3} \mathrm{~S}$ : C, 60.73; H, 4.08; N, 2.83\%. Found: C, 60.87; H, 4.05; N, $2.78 \%$.

\section{2d12 N-benzyl-4,5-bis(4-methoxyphenyl)}

thiophene-2-carboxamide (5l): Prepared from 3 and benzyl amine. Yellow powder, M.p. $60-63^{\circ} \mathrm{C}$. IR (KBr) $\mathrm{cm}^{-1}$ : 3306, 2929, 2834, 1624, 1553, 1247, 1030, 829, 795. ${ }^{1} \mathrm{H}$ NMR (400 MHz, DMSO-d 6 ): $\delta 9.09(\mathrm{t}, 1 \mathrm{H}, J=7.3 \mathrm{~Hz})$, $7.91(\mathrm{~s}, 1 \mathrm{H}), 7.35-7.33(\mathrm{~m}, 4 \mathrm{H}), 7.27-7.23$ (m, 1H), 7.21$7.17(\mathrm{~m}, 4 \mathrm{H}), 6.93-6.89(\mathrm{~m}, 4 \mathrm{H}), 4.49(\mathrm{~d}, 2 \mathrm{H}, J=5.9 \mathrm{~Hz})$, 3.76 (s, 3H), 3.75 (s, 3H). ${ }^{13} \mathrm{C}$ NMR (100MHz, DMSO-d 6$): \delta$ $160.9,159.2,158.5,141.3,139.4,137.2,136.9,130.9,130.1$, 129.8, 128.4, 127.9, 127.3, 126.9, 125.6, 114.3, 114.0, 55.2, 55.1, 42.6. ESI-MS m/z Calculated 429.1. Found: 430.1 $[\mathrm{M}+\mathrm{H}]^{+}$. Anal. Calculated for $\mathrm{C}_{26} \mathrm{H}_{23} \mathrm{NO}_{3} \mathrm{~S}: \mathrm{C}, 72.70 ; \mathrm{H}$, $5.40 ;$ N, 3.26\%. Found: C, 72.60; H, 5.36; N, 3.12\%.

2.2d13 N-(4-fluorobenzyl)-4,5-bis(4-methoxyphenyl) thiophene-2-carboxamide $(\mathbf{5 m})$ : Prepared from $\mathbf{3}$ and 4-fluorobenzyl amine. Yellow powder, M.p. $66-68^{\circ} \mathrm{C}$. IR $(\mathrm{KBr}) \mathrm{cm}^{-1}$ : 3307, 2929, 2835, 1625, 1554, 1291, 1032, 828, 795. ${ }^{1} \mathrm{H}$ NMR (400 MHz, DMSO-d 6$): \delta 9.09(\mathrm{t}, J=$ $5.9 \mathrm{~Hz}, 1 \mathrm{H}), 7.89(\mathrm{~s}, 1 \mathrm{H}), 7.39-7.36(\mathrm{~m}, 2 \mathrm{H}), 7.27-7.22$ $(\mathrm{m}, 3 \mathrm{H}), 7.21-7.15(\mathrm{~m}, 3 \mathrm{H}), 6.94-6.89(\mathrm{~m}, 4 \mathrm{H}), 4.46(\mathrm{~d}$, $J=5.9 \mathrm{~Hz}, 2 \mathrm{H}), 3.76(\mathrm{~s}, 3 \mathrm{H}), 3.75(\mathrm{~s}, 3 \mathrm{H}) .{ }^{13} \mathrm{C} \mathrm{NMR}$ $\left(100 \mathrm{MHz}, \mathrm{DMSO}_{6}\right): \delta 162.4,162.3,160.9,160.0,159.2$, $158.4,141.3,137.2,136.9,135.6,135.5,131.0,130.1$, $129.7,129.4,129.3,128.9,128.2,127.8,125.5,125.3$, 115.2, 114.9, 114.3, 114.0, 55.2, 55.1, 41.8. ESI-MS m/z Calculated 447.1. Found: $448.1[\mathrm{M}+\mathrm{H}]^{+}$. Anal. Calculated for $\mathrm{C}_{26} \mathrm{H}_{22} \mathrm{FNO}_{3} \mathrm{~S}$ : C, 69.78; H, 4.96; N, 3.13\%. Found: C, 69.46; H, 4.88; N, 3.02\%.

\section{2d14 4,5-bis(4-methoxyphenyl)-N-(4-}

(trifluoromethyl)benzyl)thiophene-2-carboxamide (5n): Prepared from 3 and 4-trifluoromethyl benzyl amine. Pale brown powder, M.p. $61-63^{\circ} \mathrm{C}$. IR (KBr) cm $\mathrm{cm}^{-1}$ : 3305, 2933, 2837, 1622, 1555, 1291, 1033, 829, 795. ${ }^{1} \mathrm{H}$ NMR (400 $\left.\mathrm{MHz} \mathrm{CDCl}_{3}\right): \delta 7.59(\mathrm{~d}, J=8.1 \mathrm{~Hz}, 2 \mathrm{H}), 7.53(\mathrm{~s}, 1 \mathrm{H}), 7.46$ $(\mathrm{d}, J=8.1 \mathrm{~Hz}, 2 \mathrm{H}), 7.22-7.20(\mathrm{~m}, 4 \mathrm{H}), 6.82-6.79(\mathrm{~m}, 4 \mathrm{H})$, $6.55(\mathrm{t}, J=6.0 \mathrm{~Hz}, 1 \mathrm{H}), 4.67(\mathrm{~d}, J=5.9 \mathrm{~Hz}, 2 \mathrm{H}), 3.79(\mathrm{~s}$, $3 \mathrm{H}), 3.78(\mathrm{~s}, 3 \mathrm{H}) .{ }^{13} \mathrm{C} \mathrm{NMR}\left(100 \mathrm{MHz}, \mathrm{CDCl}_{3}\right): \delta 162.1$, $159.6,158.9,142.9,142.3,137.8,134.8,131.5,130.4$, $130.1,130.0,129.7,128.2,128.0,125.9,125.7,125.6$, 125.5, 125.63, 125.4, 122.7, 114.1, 113.9, 55.3, 55.2, 43.4 . ESI-MS m/z Calculated 497.1. Found: 498.1 $[\mathrm{M}+\mathrm{H}]^{+}$. Anal. Calculated for $\mathrm{C}_{27} \mathrm{H}_{22} \mathrm{~F}_{3} \mathrm{NO}_{3} \mathrm{~S}$ : C, 65.18; H, 4.46; N, 2.82\%. Found: C, 64.88; H, 4.38; N, 3.02\%.

$2.2 \mathrm{~d} 15$ 4,5-bis(4-methoxyphenyl)-N-(2-(thiophen-2yl)ethyl)thiophene-2-carboxamide (5o): Prepared from 3 and thiophene-2-ethylamine. Light brown powder, M.p. $137-140^{\circ} \mathrm{C}$. IR (KBr) cm ${ }^{-1}: 3349,2934,2835,1626,1557$, 
1248, 1029, 832, 793. ${ }^{1} \mathrm{H}$ NMR (400 MHz, $\left.\mathrm{CDCl}_{3}\right): \delta 7.44$ (s, 1H), 7.23-7.21 (m, 2H), 7.18-7.15 (m, 3H), 6.97-6.95 (m, 1H), 6.88-6.87 (m, 1H), 6.83-6.79 (m, 4H), 6.17 (bs, $1 \mathrm{H}), 3.79(\mathrm{~s}, 6 \mathrm{H}), 3.74$ (quartet, $J=6.4 \mathrm{~Hz}, 2 \mathrm{H}), 3.17(\mathrm{t}, J=$ $6.5 \mathrm{~Hz}, 2 \mathrm{H}) .{ }^{13} \mathrm{C}$ NMR $\left(100 \mathrm{MHz}, \mathrm{CDCl}_{3}\right): \delta 161.9,159.6$, $158.8,142.4,141.2,137.7,135.4,131.1,130.5,130.1$, $128.3,127.2,126.0,125.5,124.1,114.1,113.9,55.3,55.2$, 41.3, 30.0. ESI-MS m/z Calculated 449.1. Found: 450.1 [M $+\mathrm{H}]^{+}$. Anal. Calculated for $\mathrm{C}_{25} \mathrm{H}_{23} \mathrm{NO}_{3} \mathrm{~S}_{2}: \mathrm{C}, 66.79 ; \mathrm{H}$, $5.16 ; \mathrm{N}, 3.12 \%$. Found: C, 69.32; H, 5.14; N, 3.56\%.

$2.2 \mathrm{e}$ General synthetic procedure for the title compounds 4,5-bis(4-hydroxyphenyl)-substituedthiophene-2-carboxamide $(\boldsymbol{6 a}-\mathbf{6 o})$ : The synthesis was similar to the synthesis of compounds 5a-5o. Using 4,5-bis(4-hydroxyphenyl)thiophene-2carboxylic acid(4) and appropriate amine as raw materials, title compounds $\mathbf{6 a - 6 0}$ were obtained. The reaction timing and yield of the product are shown in Table 1.

\section{2e1 4,5-bis(4-hydroxyphenyl)-N-propylthiophene-}

2-carboxamide (6a): Prepared from 4 and n-propyl amine. Off-white powder, M.p. $193-195^{\circ} \mathrm{C}$. IR $(\mathrm{KBr}) \mathrm{cm}^{-1}$ : 3391, 2926, 1651, 1549, 1262, 1173, 832. ${ }^{1} \mathrm{H}$ NMR (400 MHz, DMSO-d $\left.\mathrm{d}_{6}\right): \delta 8.92(\mathrm{~s}, 1 \mathrm{H}), 8.72(\mathrm{~s}, 1 \mathrm{H}), 8.52(\mathrm{t}, J$ $=7.3 \mathrm{~Hz}, 1 \mathrm{H}), 7.71(\mathrm{~s}, 1 \mathrm{H}), 7.24-7.18(\mathrm{~m}, 4 \mathrm{H}), 6.90-6.76$ $(\mathrm{m}, 4 \mathrm{H}), 3.26(\mathrm{~m}, 2 \mathrm{H}), 1.59(\mathrm{~m}, 2 \mathrm{H}), 0.88(\mathrm{t}, J=7.3 \mathrm{~Hz}, 3 \mathrm{H})$. ${ }^{13} \mathrm{C}$ NMR $\left(100 \mathrm{MHz}, \mathrm{CDCl}_{3}+\mathrm{DMSO}_{-} \mathrm{d}_{6}\right): \delta 161.1,159.3$, 158.6, 140.8, 137.6, 137.4, 130.8, 130.2, 129.8, 127.8, 125.6, 114.2, 114.1, 40.8, 22.6, 11.5. ESI-MS m/z Calculated 353.1. Found: $354.1[\mathrm{M}+\mathrm{H}]^{+}$. Anal. Calculated for $\mathrm{C}_{20} \mathrm{H}_{19} \mathrm{NO}_{3} \mathrm{~S}: \mathrm{C}, 67.97 ; \mathrm{H}, 5.42 ; \mathrm{N}, 3.96 \%$. Found: C, 67.86; $\mathrm{H}, 5.62 ; \mathrm{N}, 3.90 \%$.

\section{2e2 4,5-bis(4-hydroxyphenyl)-N-isopropylthiophene-}

2-carboxamide $(\boldsymbol{6} \boldsymbol{b})$ : Prepared from $\mathbf{4}$ and isopropyl amine. Off-white powder, M.p. $226-228^{\circ} \mathrm{C}$. IR $(\mathrm{KBr}) \mathrm{cm}^{-1}$ : 3359, 2933, 1611, 1557, 1265, 1173, 832. ${ }^{1} \mathrm{H}$ NMR (400 MHz, DMSO-d $\left.{ }_{6}\right): \delta 9.69(\mathrm{~s}, 1 \mathrm{H}), 9.51(\mathrm{~s}, 1 \mathrm{H}), 8.22(\mathrm{~d}, J=$ $7.8 \mathrm{~Hz}, 1 \mathrm{H}), 7.82(\mathrm{~s}, 1 \mathrm{H}), 7.10-7.05(\mathrm{~m}, 4 \mathrm{H}), 6.73-6.70(\mathrm{~m}$, $4 \mathrm{H}), 4.09-4.01(\mathrm{~m}, 1 \mathrm{H}), 1.17(\mathrm{~d}, J=6.6 \mathrm{~Hz}, 6 \mathrm{H}) .{ }^{13} \mathrm{C} \mathrm{NMR}$ (100 MHz, DMSO-d $\left.\mathrm{d}_{6}\right): \delta 160.6,157.9,157.0,141.5,137.5$, 137.4, 131.1, 130.5, 130.2, 126.9, 124.6, 116.0, 115.8, 41.5, 22.9. ESI-MS m/z Calculated 353.1. Found: $354.1[\mathrm{M}+$ $\mathrm{H}]^{+}$. Anal. Calculated for $\mathrm{C}_{20} \mathrm{H}_{19} \mathrm{NO}_{3} \mathrm{~S}: \mathrm{C}, 67.97 ; \mathrm{H}, 5.42$; N, 3.96\%. Found: C, 67.92; H, 5.56; N, 3.90\%.

2.2e3 N-butyl-4,5-bis(4-hydroxyphenyl)thiophene-2carboxamide (6c): Prepared from 4 and n-butyl amine. Brown powder, M.p. $256-259^{\circ} \mathrm{C}$. IR $(\mathrm{KBr}) \mathrm{cm}^{-1}: 3360$, 2929, 1608, 1563, 1252, 1177, 834. ${ }^{1} \mathrm{H}$ NMR (400 MHz, $\left.\mathrm{CDCl}_{3}+\mathrm{DMSO}_{6} \mathrm{~d}_{6}\right): \delta 8.94(\mathrm{~s}, 1 \mathrm{H}), 8.77(\mathrm{~s}, 1 \mathrm{H}), 7.45(\mathrm{~m}$, $1 \mathrm{H}), 7.09$ (s, 1H), 6.98-6.89 (m, 4H), 6.61-6.58 (m, 4H), 3.25 (quartet, $J=6.9 \mathrm{~Hz}, 2 \mathrm{H}), 1.45-1.39(\mathrm{~m}, 2 \mathrm{H}), 1.27-$ $1.21(\mathrm{~m}, 2 \mathrm{H}), 0.80(\mathrm{t}, J=7.2 \mathrm{~Hz}, 3 \mathrm{H}) .{ }^{13} \mathrm{C}$ NMR $(100 \mathrm{MHz}$, $\left.\mathrm{CDCl}_{3}+\mathrm{DMSO}_{6}\right): \delta 161.9,157.1,156.2,141.9,137.2$, $135.7,130.8,130.1,129.8,126.9,124.6,115.4,115.2,39.4$,
31.4, 19.9, 13.6. ESI-MS m/z Calculated 367.1. Found: $\mathrm{z}$ $366.2[\mathrm{M}-\mathrm{H}]^{-}$. Anal. Calculated for $\mathrm{C}_{21} \mathrm{H}_{21} \mathrm{NO}_{3} \mathrm{~S}$ : C, 68.64; H, 5.76; N, 3.81\%. Found: C, 68.54; H, 5.64; N, $3.90 \%$.

\section{2e4 N-cyclopropyl-4,5-bis(4-hydroxyphenyl)}

thiophene-2-carboxamide (6d): Prepared from 4 and cyclopropyl amine. Pale yellow powder, M.p. $167-171{ }^{\circ} \mathrm{C}$. IR (KBr) cm ${ }^{-1}: 3401,2925,2855,1609,1549,1231,1189$, 832. ${ }^{1} \mathrm{H}$ NMR (400 MHz, $\left.\mathrm{CDCl}_{3}\right): \delta 8.79(\mathrm{bs}, 2 \mathrm{H}), 7.55(\mathrm{~s}$, $1 \mathrm{H}), 7.14-7.12(\mathrm{~m}, 2 \mathrm{H}), 7.08-7.06(\mathrm{~m}, 3 \mathrm{H}), 6.76-6.74(\mathrm{~m}$, $4 \mathrm{H}), 2.88(\mathrm{~m}, 1 \mathrm{H}), 0.82-0.81(\mathrm{~m}, 2 \mathrm{H}), 0.65(\mathrm{~m}, 2 \mathrm{H}) .{ }^{13} \mathrm{C}$ NMR (100 MHz, DMSO-d $\left.{ }_{6}\right): \delta 162.7,158.9,157.5,141.9$, 137.4, 136.6, 131.4, 130.4, 130.1, 126.5, 123.8, 116.2, 115.9, 23.3, 6.2. ESI-MS m/z Calculated 351.1. Found: 352.1 [M $+\mathrm{H}]^{+}$. Anal. Calculated for $\mathrm{C}_{20} \mathrm{H}_{17} \mathrm{NO}_{3} \mathrm{~S}$ : C, 68.36; $\mathrm{H}$, 4.88 ; N, 3.99\%. Found: C, 68.56; H, 4.66; N, 3.92\%.

\section{2e5 N-cyclopentyl-4,5-bis(4-hydroxyphenyl)}

thiophene-2-carboxamide (6e): Prepared from 4 and cyclopentyl amine. Brown powder, M.p. $216-219^{\circ} \mathrm{C}$. IR (KBr) $\mathrm{cm}^{-1}: 3391,2958,2869,1611,1565,1261,1174$, 835. ${ }^{1} \mathrm{H}$ NMR (400 MHz, DMSO-d 6 ): $\delta 9.69$ (bs, $\left.1 \mathrm{H}\right), 9.51$ (bs, $1 \mathrm{H}), 8.26(\mathrm{~d}, J=7.3 \mathrm{~Hz}, 1 \mathrm{H}), 7.84(\mathrm{~s}, 1 \mathrm{H}), 7.10-7.06$ (m, 4H), 6.73-6.71 (m, 4H), 4.21-4.14 (m, 1H), 1.91-1.85 (m, 2H), 1.69-1.60 (m, 2H), 1.56-1.49 (m, 4H). ${ }^{13} \mathrm{C}$ NMR $\left(100 \mathrm{MHz}, \mathrm{DMSO}-\mathrm{d}_{6}\right): \delta 160.6,157.5,156.6,141.1,137.1$, $136.9,130.8,130.0,129.7,126.5,124.1,115.6,115.3$, 50.9, 32.2, 23.6. ESI-MS m/z Calculated 379.1. Found: $380.1[\mathrm{M}+\mathrm{H}]^{+}$. Anal. Calculated for $\mathrm{C}_{22} \mathrm{H}_{21} \mathrm{NO}_{3} \mathrm{~S}: \mathrm{C}$, 69.63; H, 5.58; N, 3.69\%. Found: C, 69.42; H, 5.62; N, $3.80 \%$.

\section{2e6 N-cyclohexyl-4,5-bis(4-hydroxyphenyl)}

thiophene-2-carboxamide (6f): Prepared from 4 and cyclohexyl amine. Brown powder, M.p. $162-164^{\circ} \mathrm{C}$. IR $(\mathrm{KBr}) \mathrm{cm}^{-1}: 3367,2930,2854,1609,1555,1258,1172$, 833. ${ }^{1} \mathrm{H}$ NMR (400 MHz, DMSO-d 6 ): $\delta 9.71(\mathrm{bs}, 1 \mathrm{H}), 9.53$ (bs, $1 \mathrm{H}), 8.20(\mathrm{~d}, J=8.0 \mathrm{~Hz}, 1 \mathrm{H}), 7.83(\mathrm{~s}, 1 \mathrm{H}), 7.09-7.05$ $(\mathrm{m}, 4 \mathrm{H}), 6.73-6.70(\mathrm{~m}, 4 \mathrm{H}), 3.71(\mathrm{~m}, 1 \mathrm{H}), 1.83(\mathrm{~m}, 2 \mathrm{H})$, $1.73(\mathrm{~m}, 2 \mathrm{H}), 1.62-1.59(\mathrm{~m}, 1 \mathrm{H}), 1.29-1.23(\mathrm{~m}, 4 \mathrm{H}), 1.13-$ $1.11(\mathrm{~m}, 1 \mathrm{H}) .{ }^{13} \mathrm{C}$ NMR $\left(100 \mathrm{MHz}, \mathrm{DMSO}-\mathrm{d}_{6}\right): \delta 160.1$, 157.5, 156.6, 141.1, 137.1, 136.9, 130.7, 130.1, 129.8, 126.5, 124.2, 115.6, 115.3, 48.4, 32.6, 25.3, 24.9. ESI-MS m/z Calculated 393.1. Found: $394.1[\mathrm{M}+\mathrm{H}]^{+}$. Anal. Calculated for $\mathrm{C}_{23} \mathrm{H}_{23} \mathrm{NO}_{3} \mathrm{~S}$ : C, 70.20; H, 5.89; N, 3.56\%. Found: C, $69.88 ; \mathrm{H}, 5.68 ; \mathrm{N}, 3.60 \%$.

\section{2e7 N-cycloheptyl-4,5-bis(4-hydroxyphenyl)}

thiophene-2-carboxamide (6g): Prepared from 4 and cycloheptyl amine. Brown powder, M.p. $150-152^{\circ} \mathrm{C}$. IR (KBr) $\mathrm{cm}^{-1}: 3370,2927,2855,1609,1554,1260,1172$, 833. ${ }^{1} \mathrm{H}$ NMR (400 MHz, DMSO-d 6 ): $\delta 9.71(\mathrm{bs}, 1 \mathrm{H}), 9.55$ (bs, $1 \mathrm{H}), 8.24(\mathrm{~d}, J=9.0 \mathrm{~Hz}, 1 \mathrm{H}), 7.84(\mathrm{~s}, 1 \mathrm{H}), 7.09-7.05$ (m, 4H), 6.72-6.70 (m, 4H), 3.96-3.87 (m, 1H), 1.88-1.81 (m, 2H), 1.69-1.63 (m, 2H), 1.57-1.54 (m, 6H), 1.52-1.44 
$(\mathrm{m}, 2 \mathrm{H}) .{ }^{13} \mathrm{C}$ NMR (100 MHz, DMSO-d 6 ): $\delta$ 159.9, 157.5, $156.6,141.1,137.1,137.0,130.7,130.1,129.8,126.5$, 124.2, 115.6, 115.3, 50.4, 34.4, 27.9, 23.9. ESI-MS m/z Calculated 407.1. Found: $408.2[\mathrm{M}+\mathrm{H}]^{+}$. Anal. Calculated for $\mathrm{C}_{24} \mathrm{H}_{25} \mathrm{NO}_{3} \mathrm{~S} \mathrm{C}, 70.73 ; \mathrm{H}, 6.18 ; \mathrm{N}, 3.44 \%$. Found: C, $70.56 ; \mathrm{H}, 6.20 ; \mathrm{N}, 3.26 \%$.

2.2e8 4,5-bis(4-hydroxyphenyl)-N-phenylthiophene2-carboxamide (6h): Prepared from $\mathbf{4}$ and aniline. Pale green powder, M.p. 236-240 ${ }^{\circ}$. IR $(\mathrm{KBr}) \mathrm{cm}^{-1}$ : 3376, 2959, 1629, 1547, 1252, 1180, 833. ${ }^{1} \mathrm{H}$ NMR (400 MHz, DMSO-d $\left.{ }_{6}\right): \delta 10.17(\mathrm{~s}, 1 \mathrm{H}), 9.75(\mathrm{~s}, 1 \mathrm{H}), 9.56(\mathrm{~s}, 1 \mathrm{H}), 8.07$ $(\mathrm{s}, 1 \mathrm{H}), 7.75(\mathrm{~d}, J=7.8 \mathrm{~Hz}, 2 \mathrm{H}), 7.38(\mathrm{t}, J=7.6 \mathrm{~Hz}, 2 \mathrm{H})$, 7.14-7.09 (m, 5H), 6.75-6.73 (m, 4H). ${ }^{13} \mathrm{C}$ NMR (100 MHz, $\left.\mathrm{CDCl}_{3}+\mathrm{DMSO}_{\mathrm{d}}\right): \delta 160.5,157.3,156.4,143.2,138.7$, $137.5,136.2,131.8,130.2,129.9,128.6,127.1,124.7$, 123.7, 120.3, 115.6, 115.4. ESI-MS m/z Calculated 387.1. Found: $388.1[\mathrm{M}+\mathrm{H}]^{+}$. Anal. Calculated for $\mathrm{C}_{23} \mathrm{H}_{17} \mathrm{NO}_{3} \mathrm{~S}$ : C, 71.30; H, 4.42; N, 3.62\%. Found: C, 71.60; H, 4.48; N, $3.56 \%$.

\section{2e9 N-(4-fluorophenyl)-4,5-bis(4-hydroxyphenyl)} thiophene-2-carboxamide (6i): Prepared from 4 and 4-fluoroaniline. Pale green powder, M.p. $226-233^{\circ} \mathrm{C}$. IR $(\mathrm{KBr}) \mathrm{cm}^{-1}: 3379,2926,1636,1509,1223,1172,831 .{ }^{1} \mathrm{H}$ NMR (400 MHz, DMSO-d $\left.)_{6}\right) \delta 10.24(\mathrm{~s}, 1 \mathrm{H}), 9.77(\mathrm{~s}, 1 \mathrm{H})$, $9.57(\mathrm{~s}, 1 \mathrm{H}), 8.04(\mathrm{~s}, 1 \mathrm{H}), 7.77-7.74(\mathrm{~m}, 2 \mathrm{H}), 7.23-7.18$ (m, 2H), 7.14-7.09 (m, 4H) 6.76-6.73 (m, 4H). ${ }^{13} \mathrm{C} \mathrm{NMR}$ $\left(100 \mathrm{MHz}, \mathrm{DMSO}-\mathrm{d}_{6}\right): \delta 160.1,158.1,157.6,157.2,142.9$, $137.9,136.7,135.6,135.6,132.4,130.5,130.4,130.3$, 126.7, 124.4, 122.5, 122.4, 116.1, 115.9, 115.7. ESI-MS m/z Calculated 405.1. Found: 406.1 $[\mathrm{M}+\mathrm{H}]^{+}$. Anal. Calculated for $\mathrm{C}_{23} \mathrm{H}_{16} \mathrm{FNO}_{3} \mathrm{~S}$ : C, 68.13; H, 3.98; N, 3.45\%. Found: C, $68.22 ; \mathrm{H}, 3.88$; N, $3.48 \%$.

2.2e10 N-(4-chlorophenyl)-4,5-bis(4-hydroxyphenyl) thiophene-2-carboxamide (6j): Prepared from 4 and 4-chloroaniline. Pale green powder, M.p. $170-174^{\circ} \mathrm{C}$. IR (KBr) $\mathrm{cm}^{-1}: 3400,2929,1638,1515,1230,1170,831 .{ }^{1} \mathrm{H}$ NMR (400 MHz, DMSO-d 6 ): $\delta 10.30$ (s, 1H), 9.77 (s, 1H), $9.57(\mathrm{~s}, 1 \mathrm{H}), 8.06(\mathrm{~s}, 1 \mathrm{H}), 7.79-7.77(\mathrm{~m}, 2 \mathrm{H}), 7.43-7.41$ $(\mathrm{m}, 2 \mathrm{H}), 7.15-7.10(\mathrm{~m}, 4 \mathrm{H})$ 6.76-6.72 (m, 4H). ${ }^{13} \mathrm{C} \mathrm{NMR}$ $\left(100 \mathrm{MHz}, \mathrm{DMSO}-\mathrm{d}_{6}\right): \delta 160.3,158.2,157.2,143.2,138.3$, $137.9,136.5,132.6,130.6,130.5,130.3,130.2,129.1$, 126.7, 124.3, 122.1, 116.7, 115.9. ESI-MS m/z Calculated 421.1. Found: $422.1[\mathrm{M}+\mathrm{H}]^{+}$. Anal. Calculated for $\mathrm{C}_{23} \mathrm{H}_{16} \mathrm{ClNO}_{3} \mathrm{~S}$ : C, 65.48; H, 3.82; N, 3.32\%. Found: C, $65.62 ; \mathrm{H}, 3.68 ; \mathrm{N}, 3.88 \%$.

2.2e11 N-(4-bromophenyl)-4,5-bis(4-hydroxyphenyl) thiophene-2-carboxamide (6k): Prepared from 4 and 4-bromoaniline. Pale green powder, M.p. $165-169^{\circ}$ C. IR $(\mathrm{KBr}) \mathrm{cm}^{-1}$ : 3401, 2928, 1636, 1515, 1231, 1170, 832. ${ }^{1} \mathrm{H}$ NMR (400 MHz, DMSO-d $\left.{ }_{6}\right): \delta 10.29(\mathrm{~s}, 1 \mathrm{H}), 9.77(\mathrm{~s}, 1 \mathrm{H})$, $9.57(\mathrm{~s}, 1 \mathrm{H}), 8.07(\mathrm{~s}, 1 \mathrm{H}), 7.74-7.71(\mathrm{~m}, 2 \mathrm{H}), 7.56-7.54$ (m, 2H), 7.15-7.10 (m, 4H) 6.76-6.72 (m, 4H). ${ }^{13} \mathrm{C}$ NMR
(100 MHz, DMSO-d $\left.\mathrm{d}_{6}\right): \delta 159.8,157.7,156.7,142.8,138.2$, $137.4,136.0,132.2,131.5,130.1,129.8,126.2,123.8$, 121.9, 115.7, 115.4. ESI-MS m/z Calculated 465.0. Found: $464.0\left[{ }_{79} \mathrm{M}^{\mathrm{Br}}-\mathrm{H}\right]^{-} ; 466.0\left[{ }_{81} \mathrm{M}^{\mathrm{Br}}-\mathrm{H}\right]^{-}$. Anal. Calculated for $\mathrm{C}_{23} \mathrm{H}_{16} \mathrm{BrNO}_{3} \mathrm{~S}$ : C, 59.24; H, 3.46; N, 3.00\%. Found: C, $59.20 ; \mathrm{H}, 3.48 ; \mathrm{N}, 2.88 \%$.

2.2e12 N-benzyl-4,5-bis(4-hydroxyphenyl)thiophene2-carboxamide $(\boldsymbol{6 l})$ : Prepared from $\mathbf{4}$ and benzyl amine. Pale green powder, M.p. $170-172^{\circ} \mathrm{C}$. IR $(\mathrm{KBr}) \mathrm{cm}^{-1}$ : 3308, 2927, 1609, 1556, 1269, 1172, 834. ${ }^{1} \mathrm{H}$ NMR (400 MHz, DMSO-d $\left.\mathrm{d}_{6}\right): \delta 9.04(\mathrm{t}, J=5.9 \mathrm{~Hz}, 1 \mathrm{H}), 7.84(\mathrm{~s}, 1 \mathrm{H})$, 7.36-7.29 (m, 4H), 7.27-7.24 (m, 1H), 7.10-7.04 (m, 4H), 6.73-6.69 (m, 4H), $4.47(\mathrm{~d}, J=5.9 \mathrm{~Hz}, 2 \mathrm{H}) .{ }^{13} \mathrm{C}$ NMR $\left(100 \mathrm{MHz}, \mathrm{DMSO}-\mathrm{d}_{6}\right): \delta 161.1,157.6,156.7,141.5,139.5$, $137.2,131.1,130.1,129.7,128.4,127.3,126.9,115.7$, 115.4, 42.5. ESI-MS m/z Calculated 401.1. Found: 400.1 $[\mathrm{M}-\mathrm{H}]^{-}$. Anal. Calculated for $\mathrm{C}_{24} \mathrm{H}_{19} \mathrm{NO}_{3} \mathrm{~S}: \mathrm{C}, 71.80 ; \mathrm{H}$, 4.77; N, 3.49\%. Found: C, 71.68; H, 4.88; N, 3.48\%.

2.2e13 N-(4-fluorobenzyl)-4,5-bis(4-hydroxyphenyl) thiophene-2-carboxamide (6m): Prepared from 4 and 4fluorobenzyl amine. Light brown powder, M.p. $248-250^{\circ} \mathrm{C}$. IR (KBr) cm $\mathrm{cm}^{-1}: 3349,2927,1608,1560,1226,1169,833$. ${ }^{1} \mathrm{H}$ NMR $\left(400 \mathrm{MHz}, \mathrm{CDCl}_{3}+\mathrm{DMSO}_{-} \mathrm{d}_{6}\right): \delta 8.99(\mathrm{~m}, 1 \mathrm{H})$ $8.81(\mathrm{~m}, 1 \mathrm{H}), 7.65(\mathrm{~m}, 1 \mathrm{H}), 7.39-7.38(\mathrm{~m}, 1 \mathrm{H}), 7.36-7.33$ $(\mathrm{m}, 2 \mathrm{H}), 7.14(\mathrm{~d}, J=8.6 \mathrm{~Hz}, 2 \mathrm{H}), 7.08(\mathrm{~d}, J=8.6 \mathrm{~Hz}, 2 \mathrm{H})$, $7.03(\mathrm{t}, J=8.71 \mathrm{~Hz}, 2 \mathrm{H}), 6.76-6.73(\mathrm{~d}, J=8.6 \mathrm{~Hz}, 4 \mathrm{H})$, $4.56(\mathrm{~d}, J=5.9 \mathrm{~Hz}, 2 \mathrm{H}) .{ }^{13} \mathrm{C}$ NMR $\left(100 \mathrm{MHz}, \mathrm{CDCl}_{3}+\right.$ DMSO- $\left.\mathrm{d}_{6}\right): \delta 163.2,162.2,160.8,157.4,156.4,142.7$, 137.6, 135.4, 135.3, 134.7, 131.4, 130.3, 129.9, 129.6, 129.5, 127.2, 124.8, 115.6, 115.5, 115.4, 115.1, 42.9. ESI-MS m/z Calculated 419.1. Found: $420.1[\mathrm{M}+\mathrm{H}]^{+}$. Anal. Calculated for $\mathrm{C}_{24} \mathrm{H}_{18} \mathrm{FNO}_{3} \mathrm{~S}$ : C, $68.72 ; \mathrm{H}, 4.33 ; \mathrm{N}, 3.34 \%$. Found: C, $68.68 ; \mathrm{H}, 4.48 ; \mathrm{N}, 3.34 \%$.

2.2e14 4,5-bis(4-hydroxyphenyl)-N-(4-(trifluoromethyl) benzyl)thiophene-2-carboxamide (6n): Prepared from 4 and 4-trifluoromethyl benzyl amine. Light brown powder, M.p. $185-189^{\circ} \mathrm{C}$. IR (KBr) cm ${ }^{-1}$ : 3361, 2928, 1627, 1556, 1256, $1176,834 .{ }^{1} \mathrm{H}$ NMR (400 MHz, $\mathrm{CDCl}_{3}+$ DMSO-d $\left._{6}\right)$ : $\delta 9.07$ (bs, 1H), 8.9 (bs, 1H), $8.16(\mathrm{bs}, 1 \mathrm{H}), 7.69(\mathrm{~s}, 1 \mathrm{H}), 7.58$ $(\mathrm{d}, J=8.2 \mathrm{~Hz}, 2 \mathrm{H}), 7.51(\mathrm{~d}, J=8.1 \mathrm{~Hz}, 2 \mathrm{H}), 7.14-7.12$ (m, 2H), 7.09-7.07 (m, 2H), 6.76-6.73 (m, 4H), $4.64(\mathrm{~d}, J$ $=5.9 \mathrm{~Hz}, 2 \mathrm{H}) .{ }^{13} \mathrm{C}$ NMR $\left(100 \mathrm{MHz}, \mathrm{CDCl}_{3}+\mathrm{DMSO}_{6}\right)$ : $\delta$ 162.4, 157.4, 156.4, 143.3, 142.8, 137.6, 135.3, 131.6, $130.3,129.9,129.3,128.9,127.9,127.2,125.5,125.3$, 125.2, 124.8, 122.8, 115.6, 115.5, 43.1. ESI-MS m/z Calculated 469.1. Found: $470.0[\mathrm{M}+\mathrm{H}]^{+}$. Anal. Calculated for $\mathrm{C}_{25} \mathrm{H}_{18} \mathrm{~F}_{3} \mathrm{NO}_{3} \mathrm{~S}: \mathrm{C}, 63.96 ; \mathrm{H}, 3.86 ; \mathrm{N}, 2.98 \%$. Found: $\mathrm{C}$, $63.88 ; \mathrm{H}, 3.68 ; \mathrm{N}, 3.00 \%$.

2.2e15 4,5-bis(4-hydroxyphenyl)- $N$-(2-(thiophen-2-yl) ethyl)thiophene-2-carboxamide (6o): Prepared from 4 and thiophene-2-ethyl amine. Off white powder, M.p. 247$249^{\circ} \mathrm{C}$. IR (KBr) cm ${ }^{-1}: 3368,2928,1610,1557,1246,1169$, 
831. ${ }^{1} \mathrm{H}$ NMR (400 MHz, DMSO-d 6 ): $\delta 9.71$ (s, 1H), 9.53 $(\mathrm{s}, 1 \mathrm{H}), 8.64(\mathrm{t}, J=5.6 \mathrm{~Hz}, 1 \mathrm{H}), 7.76(\mathrm{~s}, 1 \mathrm{H}), 7.35-7.33$ (m, 1H), 7.10-7.08 (m, 2H), 7.07-7.06 (m, 2H), 6.97-6.96 (m, $1 \mathrm{H}), 6.92-6.91(\mathrm{~m}, 1 \mathrm{H}), 6.73-6.70(\mathrm{~m}, 4 \mathrm{H}), 3.51$ (quartet, $J=7.2 \mathrm{~Hz}, 2 \mathrm{H}) 3.08(\mathrm{t}, J=7.2 \mathrm{~Hz}, 2 \mathrm{H}) .{ }^{13} \mathrm{C} \mathrm{NMR}(100$ $\left.\mathrm{MHz}, \mathrm{CDCl}_{3}+\mathrm{DMSO}_{-} \mathrm{d}_{6}\right): \delta 162.1,157.2,156.3,141.4$, $137.4,135.3,131.1,130.2,129.9,127.1,126.8,125.1$, 124.7, 123.6, 115.5, 115.3, 41.2, 29.7. ESI-MS m/z Calculated 421.1. Found: $422.1[\mathrm{M}+\mathrm{H}]^{+}$. Anal. Calculated for $\mathrm{C}_{23} \mathrm{H}_{19} \mathrm{NO}_{3} \mathrm{~S}_{2}: \mathrm{C}, 65.53 ; \mathrm{H}, 4.54 ; \mathrm{N}, 3.32 \%$. Found: $\mathrm{C}$, $65.72 ; \mathrm{H}, 4.56 ; \mathrm{N}, 3.40 \%$.

\subsection{In vitro anti-inflammatory activity (Anti-denaturation assay)}

The in vitro anti-inflammatory activity of the synthesized compounds was studied using bovine serum albumin denaturation method. ${ }^{21,22}$ In brief, increasing concentrations of the test or reference compound were incubated with $0.5 \%$ $\mathrm{w} / \mathrm{v}$ of bovine serum albumin at $37^{\circ} \mathrm{C}$ for $20 \mathrm{~min}$ and the temperature was increased to keep the samples at $57^{\circ} \mathrm{C}$ for $30 \mathrm{~min}$. After cooling to room temperature, the turbidity was measured using UV-Visible spectrophotometer at $660 \mathrm{~nm}$ following addition of phosphate buffered saline. The control represents $100 \%$ protein denaturation. The results were compared with reference drug Diclofenac sodium. The percentage inhibition of protein denaturation was calculated by using the following formula.

Percentage Inhibition $=100$

$$
\begin{aligned}
& -[\text { (optical density of test solution } \\
& \text {-optical density of product control }) \\
& \div \text { (optical density of test control) }] \times 100
\end{aligned}
$$

\subsection{Molecular docking studies}

In silico molecular docking simulation was executed using the AutoDock Tools (ADT) version 1.5.6 and AutoDock version 4.2.5.1 docking program. ${ }^{23,24}$ Three dimensional structure of COX-2 receptor was obtained from the Protein Data Bank (PDB ID: 1PXX). ${ }^{25}$ The co-crystallized ligand in the 1PXX structure was removed. The water molecules present with the crystal were deleted, the polar hydrogen atoms were added, lower occupancy residue structures were deleted, and any incomplete side chains were replaced using the ADT. Gasteiger charges were added to each atom and merged the non-polar hydrogen atoms to the protein structure. The hydrogen bond distance between donor and acceptor atoms was defined as $1.9 \AA$ with a tolerance of $0.5 \AA$, and the threshold for acceptor- hydrogen-donor angle was set to not less than $120^{\circ}$. Then, the structures were saved in PDBQT file format for further studies in ADT. Around the binding site of co-crystallised ligand, a grid box with the dimension of $60 \times$ $60 \times 60 \AA^{3}$ with $0.375 \AA$ spacing centred on $27.131,24.348$, 14.747 was created using ADT. The centre of the box was set at co-crystallised ligand centre and grid energy calculations were carried out. AutoDock docking calculation was done using default parameters and twenty docked conformations were generated for each compound. The energy of the binding interactions was estimated using genetic algorithm. The outputs were exported to PyMOL and visualised the binding modes and interactions of the compounds with amino acid residues in the active sites. ${ }^{26}$

\section{Results and Discussion}

\subsection{Chemistry}

In the present work, we synthesized a novel series of 4, 5-diarylthiophene-2-carboxamide derivatives which is shown in Scheme 1. The first step in the Scheme 1 is the Vilsmeier reaction ${ }^{27,28}$ of desoxyanisoin 1 with dimethylformamide (DMF) and phosphorous oxychloride $\left(\mathrm{POCl}_{3}\right)$ to give compound $\mathbf{2}$ in $\mathbf{7 8 \%}$ yield after recrystallization from ethanol. ${ }^{20}$ The obtained compound $\mathbf{2}$ was condensed and cyclized with 2-mercaptoacetic acid (thioglycolic acid) in the presence of potassium hydroxide to afford compound $\mathbf{3}^{29}$ in $71 \%$ yield. We studied the demethylation of compound $\mathbf{3}$ with several reactants in different reaction conditions. Treatment of compound $\mathbf{3}$ with aqueous hydrobromic acid ${ }^{30}$ or hydrobromic acid in acetic acid, ${ }^{31,32}$ gave no product. Reaction of compound 3 with boron tribromide ${ }^{33}$ ( 4 and 6 equivalents) in methylene chloride gave the product in $50 \%$ yield. Treatment of compound $\mathbf{3}$ with aluminium chloride in dichloromethane ${ }^{34}$ or benzene or toluene ${ }^{35}$ in reflux condition gave compound 4 in $30-50 \%$ yield. In our present procedure, it was found that 4 mole of aluminium chloride in chlorobenzene ${ }^{36}$ at $95-105^{\circ} \mathrm{C}$ over $3 \mathrm{~h}$ under stirring is vital for the demethylation of compound 3 to get compound $\mathbf{4}$ in good yield (88\%). The obtained compound $\mathbf{4}$ did not require any further purification.

Reaction of 4,5-diarylthiophene-2-carboxylic acid $\mathbf{3}$ and $\mathbf{4}$ with various substituted amine derivatives (alkyl, cycloalkyl, aryl, aryl alkyl and heterocyclic alkyl) in the presence of 2-(1H-Benzotriazole-1-yl)-1,1,3,3-tetramethyluronium tetrafluoroborate (TBTU) ${ }^{37-39}$ dimethylaminopyridine in acetonitrile at $25-30^{\circ} \mathrm{C}$ afforded the amides in $72-95 \%$ yields. The amides were crystallized from ethanol. The compounds were confirmed by NMR, IR, Mass spectra and X-ray single crystallography.

The structure of the synthesized compound (5j) was confirmed by various spectral techniques such as NMR, Mass and IR data. IR spectrum of the compound (5j) revealed that the band at around $3360-3300 \mathrm{~cm}^{-1}$ corresponds to the $-\mathrm{NH}$ stretching of the amide group. The 
<smiles>CC#CC1CC1CC1CC1C(=O)c1ccc(OC)cc1</smiles><smiles>CC#CC(C)C</smiles><smiles>[R9]Oc1ccc(-c2sc(C(=O)NCC)cc2-c2ccc([N+]([R])=[W])cc2)cc1</smiles>

Scheme 1. Synthesis of 4,5-diarylthiophene-2-carboxamide derivatives. Reagents and conditions: i) DMF and $\mathrm{POCl}_{3}$, at $70-75^{\circ} \mathrm{C}$ for $4 \mathrm{~h}$; ii) 2-mercapto acetic acid, $\mathrm{KOH}$, methanol and water, at reflux for $4 \mathrm{~h}$; iii) $\mathrm{AlCl}_{3}$ and chlorobenzene, at $95-105^{\circ} \mathrm{C}$ for $3 \mathrm{~h}$; iv) TBTU, dimethylaminopyridine, acetonitrile and $\mathrm{R}_{1} \mathrm{NH}_{2}\left(\mathrm{R}_{1}=\right.$ Alkyl, Cycloalkyl, Aryl, Aryl alkyl and Heterocyclic alkyl), at $25-30^{\circ} \mathrm{C}$ for 3-7 h.

band at around $\sim 1636 \mathrm{~cm}^{-1}$ corresponds to the carbonyl group of the amide moiety. The ${ }^{1} \mathrm{H}$ NMR spectrum recorded in DMSO- $\mathrm{d}_{6}$ showed twenty protons. The six protons observed at $\delta 3.75$ and at $\delta 3.76$ ppm correspond to the methoxy protons. The singlet observed at $\delta 8.11 \mathrm{ppm}$ corresponds to the thiophene ring proton. The singlet at $\delta 10.36 \mathrm{ppm}$ corresponds to $-\mathrm{NH}$ proton of the amide group. The ${ }^{13} \mathrm{C}$ NMR spectrum recorded in DMSO- $\mathrm{d}_{6}$ showed nineteen signals, in which the signals observed at $\delta 55.2 \mathrm{ppm}$ and at $\delta 55.3 \mathrm{ppm}$ confirmed the presence of methoxy carbons. The two signals at $\delta 114.1$ and at $\delta 114.4 \mathrm{ppm}$ correspond to the four phenyl ring carbons, ortho to the methoxy group. Mass spectrum acquired in positive ionization ESI mode showed the signal at $450.3 \mathrm{Da}$ which corresponds to $\left[{ }_{35} \mathrm{M}^{\mathrm{Cl}}+\mathrm{H}\right]^{+}$, confirmed the molecular mass of the compound. The chlorine isotopic peak $\left[{ }_{37} \mathrm{M}^{\mathrm{Cl}}+\right.$ $\mathrm{H}]^{+}$was also observed at $452.0 \mathrm{Da}$. In addition to the above spectral evidences discussed, the structure of synthesized compound (5j) was further confirmed with single-crystal X-ray diffraction. The crystal data was deposited at CCDC, and the CCDC No. is 1400968. 
Similarly, the structure of $\mathbf{5 b}, \mathbf{5 l}$ and $\mathbf{5 0}$ were assigned by single-crystal X-ray diffraction, deposited at CCDC and the CCDC No. are 1064110, 1404546 and 1406561 respectively. The ORTEP diagram of above crystal compounds as shown in Figures $2 \mathrm{a}-\mathrm{d}$.

\subsection{Biological Evaluation}

3.2a In vitro Anti-inflammatory activity: The antiinflammatory activity of the novel substituted 4,5-diarylthiophene-2-carboxamide derivatives were evaluated using inhibition of bovine serum albumin denaturation method and compared with standard drug diclofenac sodium. The results are summarized in Table 2.

Most of the compounds exhibited anti-inflammatory activity that is comparable to diclofenac sodium. The free acid $\mathbf{4}$ is less potent, however derivatisation of the acid to amide with $p$-halo substituted phenyl moiety (6h-k) resulted in analogues having anti-inflammatory activity comparable to the standard drug. In addition, amidic substitution with benzyl (61), fluoro benzyl (6m), $p$-trifluoromethyl benzyl (6n) or thiophene-2ethyl (6o) group resulted in less active analogues. Introduction of cyclopropyl (6d) or cyclopentyl (6e) or cyclohexyl (6f) or cycloheptyl $(\mathbf{6 g})$ amidic group resulted in compounds that are less active than the standard drug. Similar is the case with substitution of alkyl amide group 6a-c where there is a substantial loss of activity.

Interestingly, $p$-methoxy groups of the diaryl moiety compound $\mathbf{5}$ showed a marginal increase in antiinflammatory activity in comparison to $\mathbf{6}$. However, an increase in potency is not seen with the corresponding $p$-methoxy analogues of 4,5-diaryl thiophenes-2carboxamide having $p$-halo substituted phenyl amidic

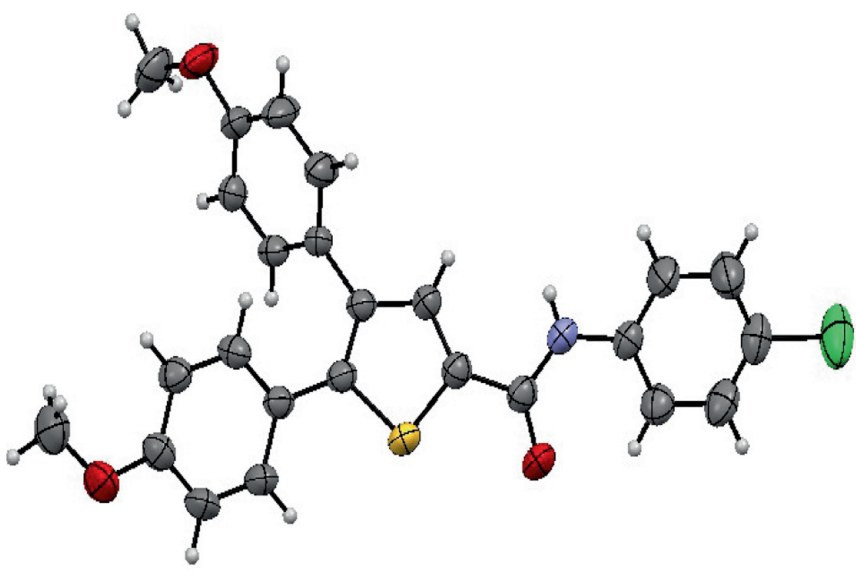

(a)

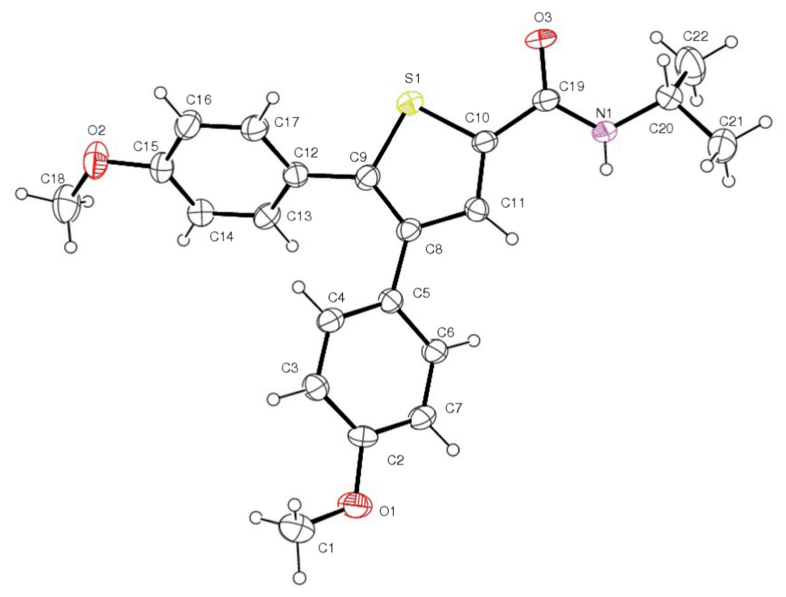

(b)

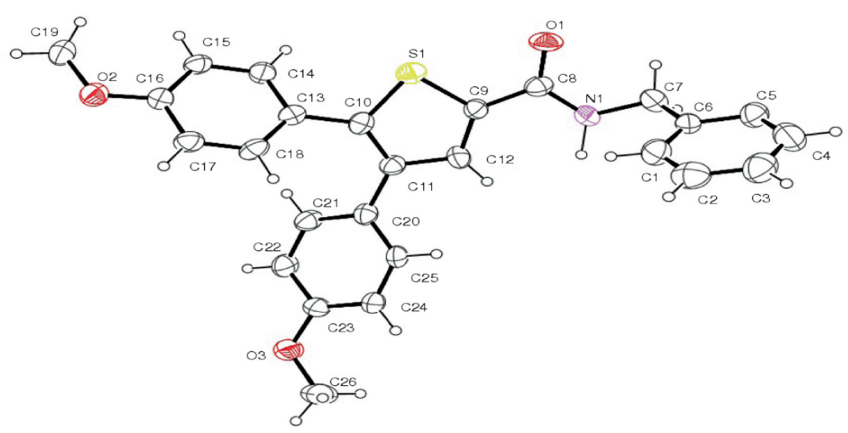

(c)

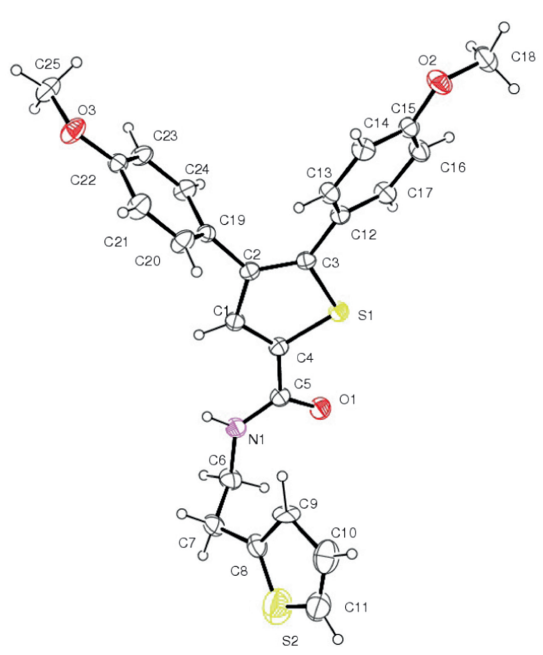

(d)

Figure 2. (a) ORTEP diagram of compound 5j [N-(4-chlorophenyl)-4, 5-bis (4-methoxyphenyl) thiophene-2-carboxamide (CCDC 1400968)]; (b) ORTEP diagram of compound 5b [N-isopropyl-4,5-bis(4-methoxyphenyl)thiophene-2-carboxamide (CCDC 1064110)]; (c) ORTEP diagram of compound 51 [N-benzyl-4,5-bis(4-methoxyphenyl)thiophene-2-carboxamide (CCDC 1404546)]; (d) ORTEP diagram of compound 5o [4,5-bis(4-methoxyphenyl)-N-(2-(thiophen-2-yl)ethyl)thiophene-2carboxamide (CCDC 1406561)]. 
Table 2. Activity (\% inhibition) and Free energy of binding (FEB) of all the synthesised compounds.

\begin{tabular}{|c|c|c|c|c|c|c|}
\hline \multirow[b]{2}{*}{ Entry } & \multirow[b]{2}{*}{ Compounds } & \multicolumn{4}{|c|}{ Activity (\% inhibition of protein denaturation) } & \multirow{2}{*}{$\begin{array}{c}\text { FEB }^{\mathrm{a}}(\mathrm{kcal} / \mathrm{mol})^{\mathrm{b}} \\
\text { COX-2 (PDBID:1PXX) }\end{array}$} \\
\hline & & $50 \mu \mathrm{g} / \mathrm{mL}$ & $100 \mu \mathrm{g} / \mathrm{mL}$ & $400 \mu \mathrm{g} / \mathrm{mL}$ & $800 \mu \mathrm{g} / \mathrm{mL}$ & \\
\hline 1 & 3 & 14.98 & 20.55 & 53.01 & 41.45 & -8.54 \\
\hline 2 & $5 \mathrm{a}$ & 7.86 & 16.46 & 24.12 & 39.26 & -9.63 \\
\hline 3 & $5 b$ & 16.42 & 24.98 & 30.48 & 46.81 & -9.9 \\
\hline 4 & $5 c$ & 8.98 & 17.86 & 28.48 & 40.96 & -10.37 \\
\hline 5 & $5 d$ & 21.09 & 21.89 & 29.24 & 45.13 & -9.75 \\
\hline 6 & $5 e$ & 18.96 & 23.78 & 43.64 & 58.95 & -11.06 \\
\hline 7 & $5 f$ & 25.75 & 34.34 & 48.58 & 62.28 & -11.53 \\
\hline 8 & $5 g$ & 11.62 & 29.61 & 48.79 & 63.12 & -9.83 \\
\hline 9 & $5 \mathrm{~h}$ & 17.24 & 26.36 & 48.88 & 64.51 & -11.11 \\
\hline 10 & $5 \mathrm{i}$ & 16.32 & 20.09 & 56.96 & 73.46 & -11.06 \\
\hline 11 & $5 \mathrm{j}$ & 17.22 & 28.36 & 53.05 & 75.28 & -10.94 \\
\hline 12 & $5 \mathrm{k}$ & 16.22 & 24.31 & 56.36 & 74.03 & -11.11 \\
\hline 13 & 51 & 19.44 & 24.37 & 42.14 & 69.78 & -11.16 \\
\hline 14 & $5 \mathrm{~m}$ & 16.61 & 17.62 & 46.93 & 68.38 & -11.08 \\
\hline 15 & $5 n$ & 16.22 & 22.34 & 44.48 & 66.52 & -10.81 \\
\hline 16 & 50 & 16.22 & 17.31 & 37.26 & 46.89 & -11.63 \\
\hline 17 & 4 & 9.84 & 16.47 & 28.13 & 30.33 & -7.96 \\
\hline 18 & $6 a$ & 7.52 & 15.46 & 24.48 & 38.77 & -9.36 \\
\hline 19 & $6 b$ & 8.86 & 16.48 & 23.11 & 36.66 & -9.6 \\
\hline 20 & $6 c$ & 9.11 & 11.31 & 22.24 & 37.26 & -9.87 \\
\hline 21 & $6 \mathrm{~d}$ & 13.74 & 14.31 & 22.65 & 33.72 & -9.39 \\
\hline 22 & $6 e$ & 7.31 & 15.72 & 28.98 & 38.16 & -10.61 \\
\hline 23 & $6 f$ & 16.05 & 16.79 & 33.29 & 42.46 & -11.16 \\
\hline 24 & $6 g$ & 12.01 & 18.84 & 30.33 & 44.21 & -9.64 \\
\hline 25 & $6 \mathrm{~h}$ & 14.28 & 22.26 & 44.82 & 65.85 & -10.88 \\
\hline 26 & $6 \mathrm{i}$ & 10.71 & 21.17 & 48.69 & 68.88 & -10.72 \\
\hline 27 & $6 \mathrm{j}$ & 13.17 & 24.98 & 47.06 & 70.72 & -11.17 \\
\hline 28 & $6 \mathrm{k}$ & 15.88 & 26.85 & 50.84 & 69.48 & -11.67 \\
\hline 29 & 61 & 10.22 & 15.62 & 24.46 & 42.41 & -10.82 \\
\hline 30 & $6 \mathrm{~m}$ & 11.28 & 16.15 & 26.43 & 46.85 & -10.62 \\
\hline 31 & $6 n$ & 12.08 & 17.01 & 22.29 & 40.19 & -10.4 \\
\hline \multirow[t]{2}{*}{32} & 60 & 7.04 & 12.65 & 25.94 & 35.71 & -10.8 \\
\hline & Std & 29.24 & 50.64 & 65.38 & 75.38 & -8.15 \\
\hline
\end{tabular}

Std = Diclofenac sodium, ${ }^{\mathrm{a}}$ Free Energy of Binding, ${ }^{\mathrm{b}}$ Calculated using Autodock 4.

group $\mathbf{5 h}-\mathbf{k}$. In contrast to $\mathbf{6 1 - n}$, there is an enhancement of activity with the methoxy analogues of 4 , 5-diarylthiophene-2-carboxamide with benzyl (5l) or $p$-fluoro substituted benzyl group (5m) or $p$-trifluoromethyl benzyl (5n) which is comparable to the standard drug. The presence of $p$-methoxy group in the case of 4,5-diarylthiophene-2-carboxamide with cyclopropyl (5d) or cyclopentyl (5e) or cyclohexyl (5f) or cycloheptyl (5g) amidic group dramatically improved the anti-inflammatory activity in comparison to corresponding $p$-OH substituted 4,5-diarylthiophene2-carboxamide. As observed with 6a-c, substitution with alkyl amide to methoxy 4,5-diarylthiophene-2carboxamide resulted in less potent analogues.

Together, these results clearly suggest that $p$ methoxy group of 4,5-diarylthiophene-2-carboxamide derivatives is advantageous in improving or retaining the anti-inflammatory spectrum. In general, alkyl amides of the 4,5-diarylthiophene-2-carboxamide has poor activity whereas the $p$-substituted benzyl or phenyl or cycloalkyl amide group has optimal activity. This indicates the requirement of bulkier substitution which would interact with active sites of the biological target. In conclusion, this study has identified a number of amidic substituents to the thiophene ring and also the methoxy substitution to the diaryl ring which is crucial for the anti-inflammatory property of 4,5diarylthiophene-2-carboxamide.

\subsection{Molecular docking studies}

To rationalize the pharmacological results, the binding mode of the potent inhibitors was investigated using AutoDock Tools (ADT) version 1.5.6 and AutoDock version 4.2.5.1 docking program. ${ }^{23,24}$ In the present 
investigation, we docked these compounds to cyclooxygenase-2 (COX-2) receptor (PDB ID: 1PXX) ${ }^{25}$ crystal structure to gain insights into the possible reasons for the anti-inflammatory activity. The cocrystallised ligand was extracted from the complex and submitted for one-ligand run calculation to verify the reproducibility of the docking calculation. Root-meansquare deviation (RMSD) value of $0.58 \AA$ of docked co-crystallised ligand with bound X-ray conformation for 1PXX, suggesting this method is valid enough to be used for docking studies of other compounds (Figure $3 \mathrm{~A})$.
In silico molecular docking studies of synthesised compounds with $1 \mathrm{PXX}$ receptor revealed that all the docked compounds bind with the receptor and exhibits free energy of binding value from -9.36 to -11.67 $\mathrm{Kcal} / \mathrm{mole}$ (Table 2). Notably, all the synthesised compounds showed higher binding affinity than the standard drug diclofenac sodium. Particularly aryl, aryl alkyl, heterocyclic derivatives of 4,5-diarylthiophene2-carboxamide showed higher binding affinity than the standard drug. All the synthesised compounds bind in the active site and the simulated conformation exhibits various interactions with different amino acids by
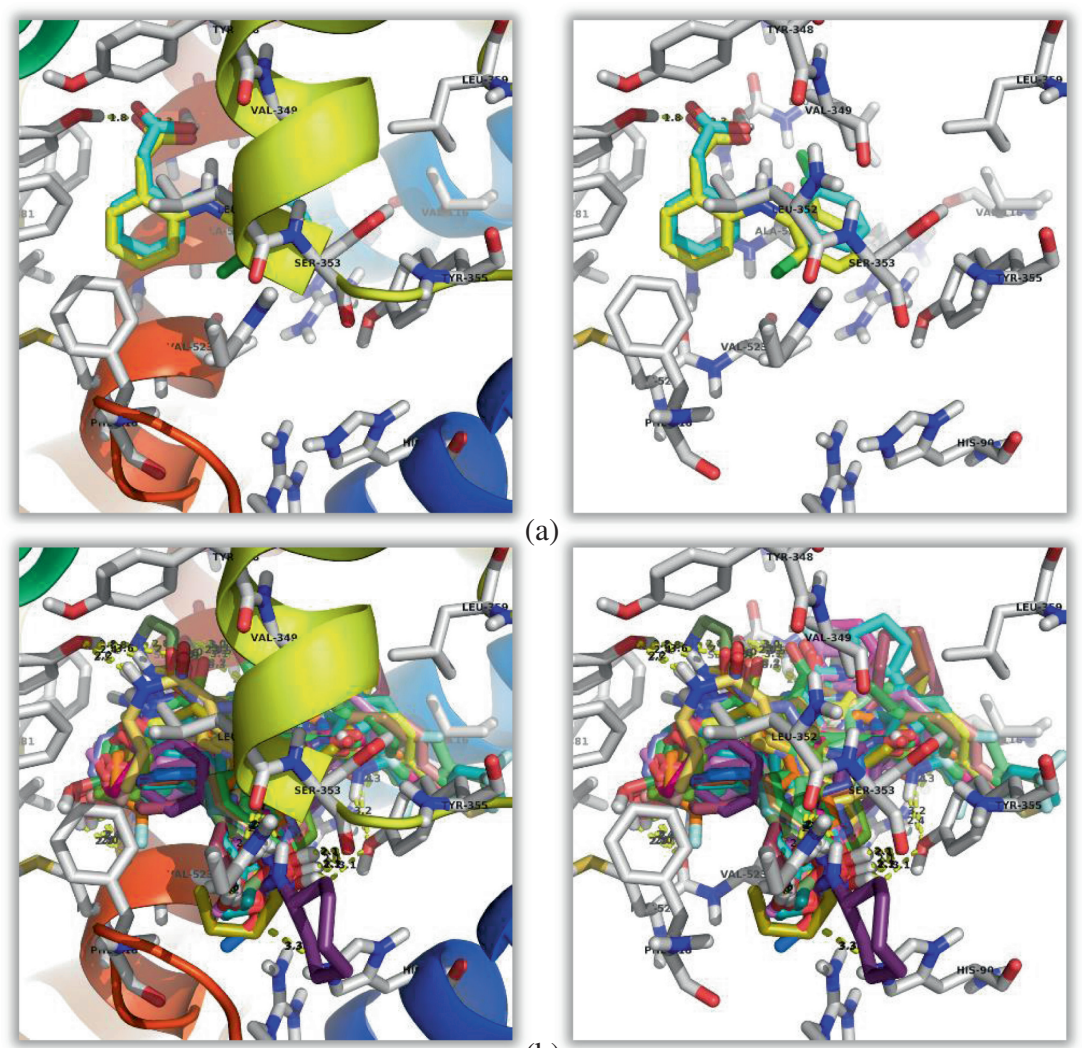

(a)
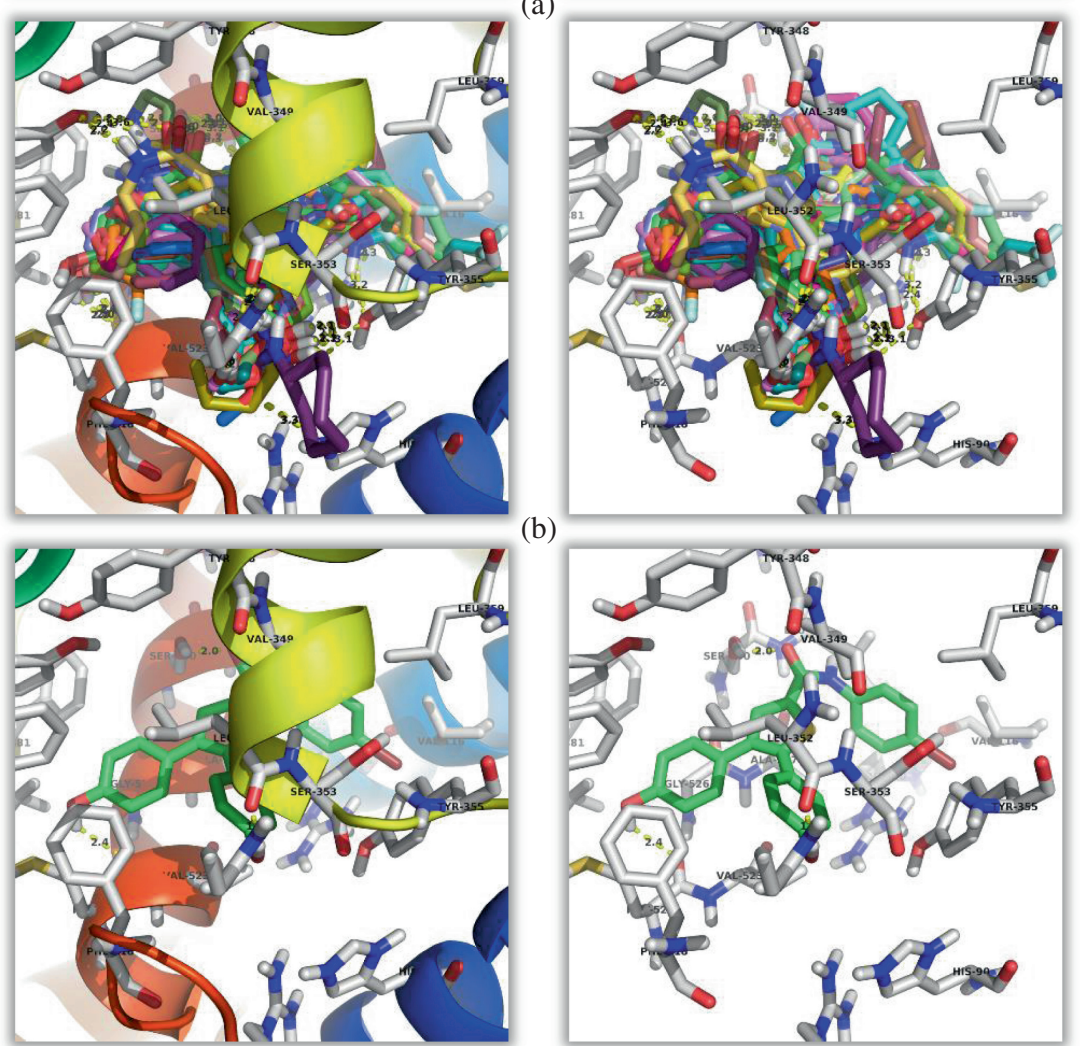

(b)

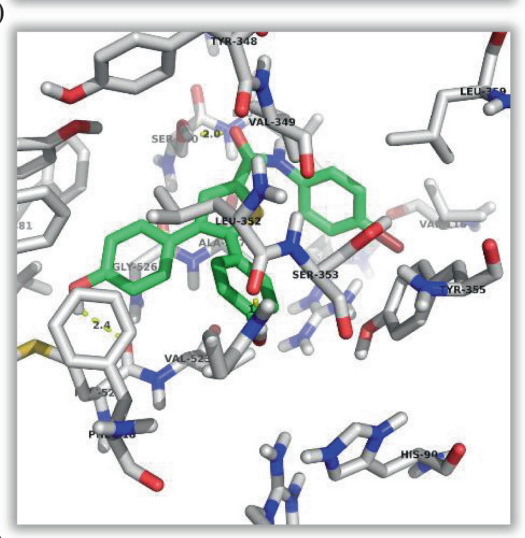

(c)

Figure 3. Molecular docking simulation with COX-2 receptor (1PXX). (a) Method validation using crystallised and docked ligand diclofenac; (b) Docking simulation of all the compounds in the active site; (c) Docking simulation of the highest binding energy compound $\mathbf{6 k}$. 
non-covalent interactions such as hydrophobic, hydrophilic, $\pi-\pi$ interaction and hydrogen bonding (Figure 3B).

Interestingly, among all the compounds docked, compound 6k exhibits very high binding with 1PXX receptor (Figure 3C) and forms three hydrogen bonds with three amino acids, namely, LEU-352, MET-522 and SER-530, which resulted in the binding energy of $-11.67 \mathrm{kcal} / \mathrm{mol}$. As shown in Figure 3C, in the compound $6 \mathbf{k}$, one of the $\mathrm{O}-\mathrm{H}$ interacts with the $\mathrm{C}=\mathrm{O}$ of LEU-352 and forms a hydrogen bond with the bond length of $1.9 \AA$. Another $\mathrm{O}-\mathrm{H}$ interacts with the $\mathrm{C}=\mathrm{O}$ of MET-522 and forms a hydrogen bond with the bond length of $2.4 \AA$. Amide $\mathrm{C}=\mathrm{O}$ interacts with the $\mathrm{O}-$ $\mathrm{H}$ of SER-530 and forms a hydrogen bond with the bond length of $2.0 \AA$. Furthermore, bromine forms polar interaction with the $\mathrm{N}-\mathrm{H}$ of ARG-120 and O-H of TYR-355 amino acid. Also, amide $\mathrm{N}-\mathrm{H}$ forms polar interaction with the $\mathrm{N}-\mathrm{H}$ of LEU-531. In addition to the polar interactions, phenyl rings exhibit hydrophobic interaction with the VAL-116, LEU-352, VAL-523, GLY-526, ALA-527, LEU-531 and LEU-350. Also, phenyl rings exhibit $\pi-\pi$ interaction with the phenyl rings of the PHE-381, TYR-385 and PHE-518.

\section{Conclusions}

In summary, we have synthesized a series of novel 4,5diarylthiophene-2-carboxamide compounds containing alkyl, cycloalkyl, aryl, aryl alkyl, and heterocyclic alkyl moieties. These compounds were characterized and evaluated for their in vitro anti-inflammatory activities. Among the novel compounds, the inhibition of bovine serum albumin denaturation assay revealed that the aryl and aryl alkyl derivatives of 4,5-diarylthiophene-2-carboxamide showed anti-inflammatory activity comparable to the standard drug diclofenac sodium, whereas alkyl and cycloalkyl amide derivatives showed less activity. From the docking studies, aryl, aryl alkyl and heterocyclic derivatives of 4,5-diarylthiophene2-carboxamide showed higher binding energy than the standard drug. Particularly, the compound (6k) N(4-bromophenyl)-4,5-bis(4-hydroxyphenyl)thiophene2-carboxamide showed high free energy binding of $-11.67 \mathrm{kcal} / \mathrm{mol}$. Further studies are in progress to improve the biological activities of 4,5-diarylthiophene-2-carboxamide derivatives.

\section{Supplementary Information (SI)}

The characterization of the compounds $\mathbf{2}, \mathbf{3}, \mathbf{4}, \mathbf{5 a}, \mathbf{5 b}, \mathbf{5} \mathbf{c}$, 5d, 5e, 5f, 5g, 5h, 5i, 5j, 5k, 5l, 5m, 5n, 5o, 6b, 6c, 6d, 6e, 6f, 6g, 6h, 6i, 6k, 61, 6m, 6n and 60 using ${ }^{1} \mathrm{HNMR},{ }^{13} \mathrm{CNMR}$,
IR and Mass data (Figures S1-S124) are given in the supporting information. Supplementary Information is available at www.ias.ac.in/chemsci.

\section{Acknowledgements}

The authors are thankful to the management of Orchid Pharma Limited, Chennai 600 119, India and Ramakrishna Mission Vivekananda College, Chennai 600 004, India for providing the required facilities.

\section{References}

1. Vane J R 1971 Inhibition of prostaglandin synthesis as a mechanism of action for aspirin-like drugs Nature $\mathbf{2 3 1}$ 232

2. Reitz D B and Isakson P C 1995 Cyclooxygenase-2 inhibitors Curr. Pharm. Design 1211

3. Gans K R, Galbraith W, Roman R J, Haber S B, Kerr J S, Schmidt W K, Smith C, Hewes W E and Ackerman N R 1990 Anti-inflammatory and safety profile of DuP 697, a novel orally effective prostaglandin synthesis inhibitor J. Pharmacol. Exp. Ther. 254180

4. Vane J R, Bakhle Y S and Botting R M 1998 Cyclooxygenases 1 and 2 Annu. Rev. Pharmacol. 3897

5. Masferrer J L, Seibert K, Zweifel B and Needleman P 1992 Endogenous glucocorticoids regulate an inducible cyclooxygenase enzyme P. Natl. Acad. Sci. U.S.A. 89 3917

6. Masferrer J L, Reddy S T, Zweifel B S, Seibert K, Needleman P, Gilbert R S and Herschman H R 1994 In vivo glucocorticoids regulate cyclooxygenase- 2 but not cyclooxygenase- 1 in peritoneal macrophages $J$. Pharmacol. Exp. Ther. 2701340

7. Tanaka A, Motoyama Y and Takasugi H 1994 Studies on Anti-platelet Agents. IV. A Series of 2-Substituted 4, 5-Bis(4-methoxyphenyl)pyrimidines as Novel Antiplatelet Agents Chem. Pharm. Bull. 421828

8. Tseng C H, Chen Y L, Chung K Y, Cheng C M, Wang $\mathrm{C} \mathrm{H}$ and Tzeng C C 2009 Synthesis and antiproliferative evaluation of 6-arylindeno[1,2-c]quinoline derivatives Bioorgan. Med. Chem. 177465

9. Gadad A K, Palkar M B, Anand K, Noolvi M N, Boreddy T S and Wagwade 2008 Synthesis and biological evaluation of 2-trifluoromethyl/sulfonamido-5,6diaryl substituted imidazo[2,1-b]-1,3,4-thiadiazoles: A novel class of cyclooxygenase-2 inhibitors Bioorgan. Med. Chem. 16276

10. Palkar M B, Noolvi M N, Maddi V S, Ghatole M and Nargund L G 2011 Synthesis, spectral studies and biological evaluation of a novel series of 2substituted-5,6-diarylsubstituted imidazo(2,1-b)-1,3,4thiadiazole derivatives as possible anti-tubercular agents Med. Chem. Res. 211313

11. Malik J K, Soni H and Singhai A K 2013 Synthesis, characterization and evaluation for antifungal activity of substituted diaryl imidazo $[2,1, \mathrm{~b}]$-benzothiazole $J$. Pharm. Res. 739 
12. Krohn K, Kulikowski K and Leclercq G 1989 Diethylstilbestrol-linked cytotoxic agents Synthesis and binding affinity for estrogen receptors J. Med. Chem. 32 1532

13. Klose W and Schwarz K 1982 Nichtsteroidale entzündungshemmer. 13 Bis-(4-methoxyphenyl)-2-arylthiopyrimidine J. Heterocycl. Chem. 191165

14. Szmuszkovicz J, Glenn E M, Heinzelman R V, Hester J B Jr. and Youngdale G A 1966 Synthesis and anti-inflammatory activity of 2,3-bis(p-methoxyphenyl)indole and related compounds J. Med. Chem. 9 527

15. Thomas E W, Nishizawa E E, Zimmermann D C and Williams D J 1985 Synthesis and platelet aggregation inhibitory activity of 4,5-bis(substituted)-1,2,3thiadiazoles J. Med. Chem. 28442

16. Marnett L J and Kalgutkar A S 1999 Cyclooxygenase 2 inhibitors: discovery, selectivity and the future Trends Pharmacol. Sci. 20465

17. Zarghi A, Rao P N P and Knaus E E 2007 Design and synthesis of new rofecoxib analogs as selective cyclooxygenase-2 (COX-2) inhibitors: Replacement of the methanesulfonyl pharmacophore by a N-acetylsulfonamido bioisostere J. Pharm. Pharm. Sci. 10159

18. Pinto D J P, Batt D G, Pitts W J, Petraitis J J, Orwat M J, Wang S, Jetter J W, Sherk S R, Houghton G C, Copeland R A, Covington M B, Trzaskos J M and Magolda R L 1999 Terphenyl cyclooxygenase-2 (COX-2) inhibitors: Optimization of the central ring and o-biphenyl analogs Bioorgan. Med. Chem. Lett. 9919

19. Pinto D J P, Copeland R A, Covington M B, Pitts W J, Batt D G, Orwat M J, Lam G N, Joshi A, Chan Y-C, Wang S, Trzaskos J M, Magolda R L and Kornhauser D M 1996 Chemistry and pharmacokinetics of diarylthiophenes and terphenyls as selective COX-2 inhibitors Bioorgan. Med. Chem. Lett. 6 2907

20. Wierzbicki M, Sauveur F, Bonnet J and Tordjman C 1998 Thiophene compounds U.S. Patent 5705525

21. Mizushima Y and Kobayashi M 1968 Interaction of antiinflammatory drugs with serum proteins, especially with some biologically active proteins J. Pharm. Pharmacol. 20169

22. Rajadurai R, Padmanabhan R and Ananthan S 2013 Synthesis and biological evaluation of diamide derivatives of (S)-BINOL and biphenyl as potential antiinflammatory/anti-arthritic agents Med. Chem. Res. 22 4164

23. Sanner M F 1999 Python: A programming language for software integration and development J. Mol. Graph. Model 1757

24. Morris G M, Huey R, Lindstrom W, Sanner M F, Belew R K, Goodsell D S and Olson A J 2009 AutoDock4 and AutoDockTools4: Automated docking with selective receptor flexibility J. Comput. Chem. 30 2785

25. Kiefer J R, Rowlinson S W, Prusakiewicz J J, Pawlitz J L, Kozak K R, Kalgutkar A S, Stallings W C, Marnett L J and Kurumbail R G 2003 Crystal structure of
Diclofenac bound to the cyclooxygenase active site of COX-2. doi: 10.2210/pdb1pxx/pdb

26. Lill M A and Danielson M L 2010 Computer-aided drug design platform using PyMOL J. Comput. Aided Mol. Des. 2513

27. Tordjman C, Sauveur F, Droual M, Briss S, Andre N, Bellot I, Deschamps C and Wierzbicki M 2003 Synthesis of the butanamide derivative $S$ 19812, a new dual inhibitor of cyclooxygenase and lipoxygenase pathways Arzneimittel-Forschung $\mathbf{5 3} 774$

28. Wang Z, Yang Q, Bai Z, Sun J, Jiang X, Song H, Wu Y and Zhang W 2015 Synthesis and biological evaluation of 2,3-diarylthiophene analogues of combretastatin A-4 MedChemComm 6971

29. Kvitko I Y 1969 Synthesis of derivatives of thieno[2,3c]pyrazole and thieno[2,3-d]thiazoline Chem. Heterocycl. Comp. 5567

30. Paolo C and Flavio V 1984 New derivatives of 1,2,3,4-tetrahydronaphthalene, process for their preparation and associated pharmaceutical compositions G.B. Patent 2123410A

31. Long L and Burger A 1941 Structural models of cortin compounds in the naphthalene series J. Org. Chem. 6 852

32. Przybyl A K, Flippen-Anderson J L, Jacobson A E and Rice K C 2003 Practical and High-Yield Syntheses of Dihydromorphine from Tetrahydrothebaine and Efficient Syntheses of (8S)-8-Bromomorphide J. Org. Chem. 682010

33. McOmie F W and West D W 1969 3,3'-Dihydroxybiphenyl Organic Syntheses 4950

34. Negi A, Chattopadhyay S, Srivastava S and Bhattacharya A 2006 Process for regioselective demethylation of p-methoxy group in phenolic ester and diaryl ketone moieties U.S. Patent 0052463

35. Chiesi P, Ventura P, Servadio V, Del C M, De F R and Amari G 1995 A process for the preparation of 5,6dihydroxy-2-amino-1,2,3,4-tetrahydronaphthalene derivatives WO Patent 029 147A1

36. Kobayashi K, Shimizu H, Sasaki A and Suginome H 1993 Photoinduced molecular transformations. 140. New one-step general synthesis of naphtho[2,3-b]furan4,9-diones and their 2,3-dihydro derivatives by the regioselective $[3+2]$ photoaddition of 2-hydroxy1,4-naphthoquinones with various alkynes and alkenes: Application of the photoaddition to a two-step synthesis of maturinone J. Org. Chem. 584614

37. Al-Tel T H, Al-Qawasmeh R A and Zaarour R 2011 Design, synthesis and in vitro antimicrobial evaluation of novel Imidazo[1,2-a]pyridine and imidazo[2,1-b] $[1,3]$ benzothiazole motifs European J. Med. Chem. 46 1874

38. Kolczewski S, Roche O, Steward L, Wichmann J and Woltering T 2010 6-substituted benzoxazines as 5-ht-5a receptor antagonists WO Patent 026 110A3

39. Balalaie S, Mahdidoust $M$ and Eshaghi-Najafabadi R 2007 2-(1H-benzotriazole-1-yl)-1,1,3,3-tetramethyluronium tetrafluoroborate as an efficient coupling reagent for the amidation and phenylhydrazation of carboxylic acids at room temperature J. Iran. Chem. Soc. 4364 